Article

\title{
A Novel Melt-Dispersion Technique for Simplistic Preparation of Chlorpromazine-Loaded Polycaprolactone Nanocapsules
}

\author{
Thiresen Govender ${ }^{1}$, Yahya E. Choonara ${ }^{1}$, Pradeep Kumar ${ }^{1}$, Lisa C. du Toit ${ }^{1}$, Girish Modi ${ }^{2}$, \\ Dinesh Naidoo ${ }^{3}$ and Viness Pillay ${ }^{1, *}$
}

1 Wits Advanced Drug Delivery Platform Research Unit, Department of Pharmacy and Pharmacology, School of Therapeutic Sciences, Faculty of Health Sciences, University of the Witwatersrand, Johannesburg, 7 York Road, Parktown 2193, South Africa;

E-Mails: thiresen.govender@students.wits.ac.za (T.G.); yahya.choonara@wits.ac.za (Y.E.C.); pradeep.kumar@wits.ac.za (P.K.); lisa.dutoit@wits.ac.za (L.C.D.T.)

2 Department of Neurology, Division of Neurosciences, Faculty of Health Sciences, University of the Witwatersrand, Johannesburg, 7 York Road, Parktown 2193, South Africa; E-Mail: gmodicns@mweb.co.za

3 Department of Neurosurgery, Division of Neurosciences, Faculty of Health Sciences, University of the Witwatersrand, Johannesburg, 7 York Road, Parktown 2193, South Africa; E-Mail: dineshnaidoo@yahoo.com

* Author to whom correspondence should be addressed; E-Mail: viness.pillay@wits.ac.za; Tel.: +27-11-717-2274; Fax: +27-86-517-6890.

Academic Editor: Cyrille Boyer

Received: 20 April 2015 / Accepted: 8 June 2015 / Published: 19 June 2015

Abstract: The aim of this study was to design, synthesize and optimize chlorpromazine
hydrochloride (CPZ)-loaded, poly- $\varepsilon$-caprolactone (PCL) based nanocapsules, intended for
site specific delivery to the frontal lobe, using a novel melt-dispersion technique that is
non-arduous, inexpensive and devoid of any hazardous organic solvents. Experimental trials
using a central composite design were performed on 13 statistically derived formulations of
various combinations of PCL (1000-3000 mg) and Polysorbate $80(2 \%-5 \% \mathrm{v} / \mathrm{v})$ on the
physicochemical and physicomechanical properties and interactive effects on PCL
nanocapsule formulation. Differential scanning calorimetry (DSC), Temperature modulated
differential scanning calorimetry (TMDSC) and Fourier transform infrared spectroscopy
(FTIR) revealed that there was no thermodegardation of the constituents utilized in the melt
dispersion technique. Nanocapsule yields achieved were very high however entrapment of 
$\mathrm{CPZ}$ proved to be relatively low due to the highly hydrophilic nature of $\mathrm{CPZ}$ and the processing of the nanocapsules post synthesis. Nanocapsule sizes were in the nanotherapeutic range and varied from $132.7 \pm 6.8 \mathrm{~nm}$ to $566.6 \pm 5.5 \mathrm{~nm}$. Zeta potential ranged from $15.1 \pm 0.65 \mathrm{mV}$ to $28.8 \pm 0.84 \mathrm{mV}$ revealing capsules that were of incipient to moderate stability. Transmission electron microscopy revealed nanocapsules that were spherical shape, well individualized with a moderate degree of flocculation. In vitro CPZ release was biphasic for all formulations with an initial burst release followed by pseudo-steady controlled release over 30 days. The cytotoxicity of the optimized nanocapsule system on a PC12 neuronal cell line proved to be minimal. Following incorporation of the optimized nanocapsules within a polymeric membrane, in vivo implantation of the device in a New Zealand Albino rabbit model proved the efficacy of the system in achieving prolonged more targeted CPZ levels to the brain. Extensive in vitro testing and optimization and preclinical evaluation supports the application for the use and feasibility of the CPZ-loaded, PCL based nanocapsules for the long-term management of certain psychotropic disorders where the benefits of nanotechnology can be exploited.

Keywords: neurotherapy; chlorpromazine hydrochloride; poly- $\varepsilon$-caprolactone; Polysorbate 80; melt-dispersion technique; nanocapsules; central composite design

\section{Introduction}

Conventional treatment for psychosis and other neurological diseases has generally proved inadequate, mainly due to the restrictive blood-brain barrier (BBB). The systemic delivery of antipsychotic drugs results in minimal transport of these agents across the BBB and results in therapeutic failure [1-6]. Therapeutic failure of the majority of neurotherapeutic drugs relies on the fact that these drugs are only transported minimally across the BBB. Apart from this, the lack of innovation in product development to counteract these challenges is also a major contributing factor to a poor therapeutic outcome $[3,7]$.

Nanotechnology provides many promising solutions to impediments arising from conventional neurotherapy. One of the major advantages of using nano-carriers is the fact that modification of the drug, intended for central nervous system (CNS) delivery, is not required. Furthermore, surface modification of these diminutive capsules may allow for brain entry of the drug molecules after an intravenous dose. This is achieved by receptor mediated transport [8]. A number of studies have established the successful nano-encapsulation of antipsychotic drugs in order to optimize neurotherapy of psychotropic disorders [9-13].

Benvegnú and co-workers (2011) successfully incorporated the antipsychotic drug, haloperidol into polysorbate nanocapsules. Extensive in vivo testing on the Wistar rat model proved that the method was feasible in terms of improving the efficacy of haloperidol with antipsychotic effects of the drug being maintained for longer periods of time when compared to free haloperiodol. In addition, the acute and sub-acute extra-pyramidal motor disorders were also significantly reduced when compared to the administration of free haloperidol [11]. Muthu and co-workers 2009 synthesized risperidone-loaded, poly(D,L-lactide-co-glycolide) (PLGA) nanocapsules that were matricized in a thermo-responsive 
in situ gel and administered subcutaneously in the Swiss albino mouse model. The antipsychotic effects of risperidone as well the marked lack of extra-pyramidal side effects were demonstrated by the system, thus confirming its success [10]. A similar study by Silva and co-workers 2011, investigated the potential of entrapping risperidone in solid lipid nanocapsules (SLN), intended for oral administration. Results revealed highly stable risperidone-loaded SLNs with negligible toxicity when tested with Caco-2 cells using the (4,5-dimethylthiazol-2-yl)2,5-diphenyl-tetrazolium bromide (MTT) assay [13].

With regard to the design of nanocapsules in optimizing therapeutic efficacy and success, various parameters need to be considered. Factors such as lipophilicity, polarity, nanocapsule size, drug entrapment and drug release kinetics are all relevant. Popular methods for the design and development of nanocapsules include; the solvent evaporation method, spontaneous emulsification/solvent diffusion method, salting out/emulsification-diffusion method, supercritical fluid technology, ionic gelation and polymerization of monomers techniques [14-16]. Most of these methods prove unsatisfactory and are related to many shortcomings such as; the inability to scale-up for large-scale pilot production, limited solubility of polymers in supercritical fluids and most of all, toxicity of using organic solvents. Apart from being environmentally hazardous, organic solvent impurities may remain in the nanocapsule post-synthesis and become physiologically dangerous and may even cause degradation of the active within the polymer matrix [14].

This study uses this principle of adaptation and implements a melt dispersion technique for the preparation of CPZ-loaded nanocapsules taking into consideration the chemical and molecular properties of the polymer to be used, poly- $\varepsilon$-caprolactone (PCL) and the active water-soluble model antipsychotic drug Chlorpromazine (CPZ). Entrapment within the nanocapsules would control its release of the entrapped drugand enhance site-specific delivery, thereby reducing dosing amount and frequency and minimizing systemic side effects. PCL is a hydrophobic, semi-crystalline, thermoplastic polymer with five non-polar methylene groups in its repeating unit and a relatively polar ester group. In physiological conditions, PCL undergoes very slow biodegradation compared to other biodegradable polyesters (e.g., polyglycolic acid, poly-L-lactide and their copolymers) by hydrolysis of its ester linkages and is biocompatible with surrounding tissues. This has sparked widespread interest in the polymer for use in long-term drug delivery systems lasting greater than a year [17-19]. The use of PCL nanocapsules to successfully entrap a variety of pharmacological actives to improve their therapeutic efficacy has been well documented. Some of these actives include but are not limited to; anti-cancer drugs, anti-inflammatories, beta blockers, anti-infective agents, insulin, immune modulators, proteins and psychotropic agents [17,19]. The majority of PCL nano-carriers are synthesized by nanoprecipitation, solvent displacement and solvent evaporation [19]. As previously discussed, these methods utilize hazardous and potentially physiologically dangerous organic solvents. The aim of this study was to design, synthesize and optimize CPZ-loaded, PCL based nanocapsules using a melt-dispersion technique that is non-arduous, inexpensive and devoid of any hazardous organic solvents, thereby eliminating the limitations of conventional nanocapsule synthesis, in particular, PCL-based nanocapsule synthesis. The study takes advantage of the thermoplastic nature of PCL which allows it to become soft and pliable once heated passed its melting point and then subjected to extreme shear whilst cooling to room temperature, thereby reforming the PCL and maintaining its integrity. From a formulatory aspect, Polysorbate 80 was included in the nanocapsule system for its stabilizing and cryoprotectant properties, thus counteracting agglomeration of the PCL nanoparticles (e.g., on centrifugation) and as a cryoprotectant to prevent freezing damage to the PCL 
polymer on lyophilisation of the nanoparticulate suspension. Mechanistically, Polysorbate 80 has been demonstrated to enhance endocytosis across the BBB via enhanced adsorption of the plasma apolipoprotein E (Apo E) on the surface of these particles. The Apo E assists in the transport of lipids into the brain by the low-density lipoprotein (LDL) receptors [8].

Following optimization, the cytotoxicity of the optimized nanocapsule system on a neuronal PC12 cell line was ascertained. The optimized nanocapsules developed were subsequently incorporated within an implantable polymeric membrane system and implanted into the frontal lobe of the brain of the New Zealand Albino rabbit model with evaluation of CPZ levels locally and systemically compared to intramuscular CPZ administration; as well as histomorphological evaluation of the effect of the implanted system on surrounding brain tissue.

\section{Experimental Section}

\subsection{Materials}

Poly- $\varepsilon$-caprolactone (PCL), chlorpromazine hydrochloride, Glycerol B.P., Polysorbate 80, hexamethylenediamine $(\mathrm{HMD})\left(M_{\mathrm{w}}=116.2 \mathrm{~g} / \mathrm{mol}\right)$, sebacoyl chloride $(\mathrm{SC})\left(M_{\mathrm{w}}=239.1 \mathrm{~g} / \mathrm{mol}\right)$, anhydrous $n$-hexane, ethylcellulose (EC), dialysis tubing, anhydrous potassium bromide, isoniazid (INH), and anhydrous sodium hydroxide pellets were purchased from Sigma Aldrich (St. Louise, MO, USA). De-ionized water was obtained from a Milli-Q water purification system (Milli-Q, Millipore, Billerica, MA, USA). All solvents utilized for UPLC-UV detection were of UPLC (Ultra-performance liquid chromatography) grade and other reagents were of analytical grade and used as purchased. The CytoTox96 ${ }^{\circledR}$ Non-Reactive Cytotoxic Assay which measures cell viability was purchased from Promega Corporation (Madison, WI, USA). Mammalian Pheochromocytoma (PC12) neuronal cells were purchased from the Health Science Research Resources Bank (HSRRB, Osaka, Japan).

\subsection{Construction of a Face-Centered Central Composite Design}

A central composite design was used to determine the direct additive effects of the study variables on the physicochemical and physicomechanical properties and interactive effects on polycaprolactone nanocapsule formulation (Table 1). Experimental trials were performed on 13 statistically derived formulations of various combinations of polymers and stabilizers. PCL (1000-3000 mg) and Polysorbate $80(2 \%-5 \% v / v)$ were selected as the independent formulation variables. Nanocapsule size, zeta potential and drug entrapment efficiency were selected as the formulation responses. A statistical model incorporating interactive and polynomial terms was utilized to evaluate the responses. Response surface plots were constructed to visually represent the influence of the stabilizer and polymeric concentrations on the nanocapsule size, zeta potential as well as chlorpromazine hydrochloride encapsulation efficiency. As a control, non-drug loaded nanocapsules were also prepared and assessed. 
Table 1. Face-centered central composite design template with statistically generated formulations.

\begin{tabular}{cccccc}
\hline Formulation No. & $\begin{array}{c}\text { PCL } \\
(\mathbf{m g})\end{array}$ & $\begin{array}{c}\text { Polysorbate 80 } \\
(\boldsymbol{\%} \mathbf{v} / \boldsymbol{v})\end{array}$ & $\begin{array}{c}\text { Glycerol } \\
(\mathbf{m L})\end{array}$ & $\begin{array}{c}\text { Stirring Speed } \\
(\mathbf{r p m})\end{array}$ & $\begin{array}{c}\mathbf{C P Z} \\
(\boldsymbol{\%} \boldsymbol{w} / \boldsymbol{v})\end{array}$ \\
\hline 1 & 2000 & 3.5 & 65 & 1500 & 5 \\
2 & 3000 & 5 & 65 & 1500 & 5 \\
3 & 3000 & 2 & 65 & 1500 & 5 \\
4 & 2000 & 3.5 & 65 & 1500 & 5 \\
5 & 1000 & 3.5 & 65 & 1500 & 5 \\
6 & 3000 & 3.5 & 65 & 1500 & 5 \\
7 & 2000 & 3.5 & 65 & 1500 & 5 \\
8 & 1000 & 2 & 65 & 1500 & 5 \\
9 & 2000 & 2 & 65 & 1500 & 5 \\
10 & 2000 & 5 & 65 & 1500 & 5 \\
11 & 2000 & 3.5 & 65 & 1500 & 5 \\
12 & 1000 & 5 & 65 & 1500 & 5 \\
13 & 2000 & 3.5 & 65 & 1500 & 5 \\
\hline
\end{tabular}

\subsection{Preparation of the PCL Nanocapsules}

Nanocapsules were prepared by a novel hot melt dispersion technique according to the design template in Table 1 and are outlined in Figure 1.

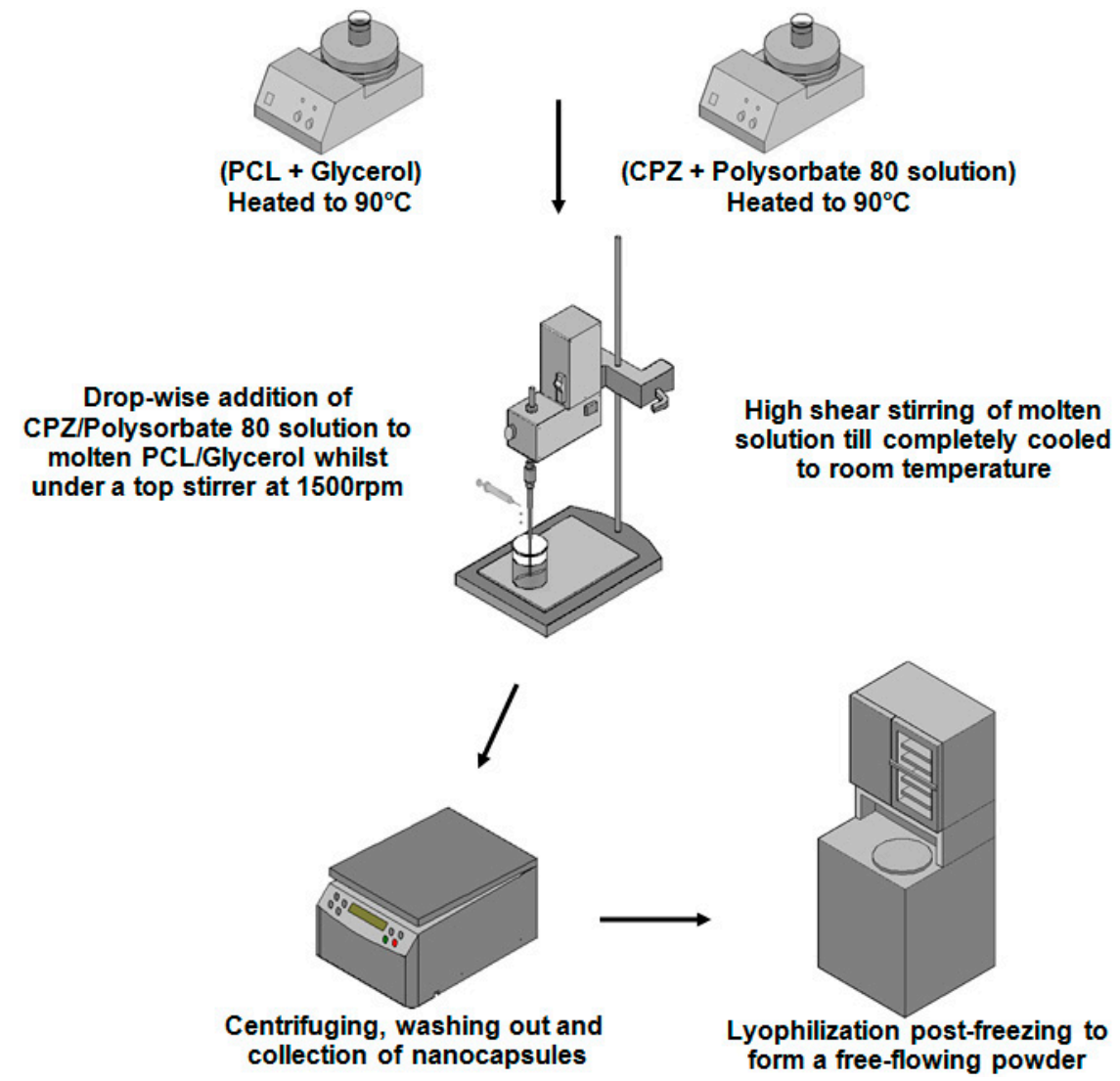

Figure 1. Schematic depicting the synthesis process of the chlorpromazine hydrochloride (CPZ)-loaded, poly- $\varepsilon$-caprolactone (PCL) based nanocapsules by the novel melt-dispersion technique. 
PCL (1000-3000 mg) was firstly dispersed in 65mL of glycerol and this was subjected to heating to $90{ }^{\circ} \mathrm{C}$ and magnetic stirring at $200 \mathrm{rpm}$ while on a heat modulated magnetic stirrer. A $5 \% \mathrm{w} / \mathrm{v}$ aqueous $\mathrm{CPZ}$ solution containing Polysorbate 80 was also heated to $90^{\circ} \mathrm{C}$. Following adequate melting of PCL in the glycerol melting base, the molten solution was transferred to a high speed, high shear top stirrer and made to rotate at $1500 \mathrm{rpm}$. This was followed by the drop-wise addition of the heated CPZ (5-15 mL), maintaining the polymer:drug ratio at 4:1, and Polysorbate 80 solution to the molten solution. The solution was allowed to stir at $1500 \mathrm{rpm}$ on the top stirrer for $45 \mathrm{~min}$ until cooled to room temperature. Nanocapsules were allowed to cure for $15 \mathrm{~min}$ and were processed by centrifuging at $3000 \mathrm{rpm}$ for $10 \mathrm{~min}$, removing the supernatant, re-dispersing in de-ionized water, with repetition of this centrifugation cycle three times. Due to the high viscosity of glycerol, de-ionized water was added to the nanocapsule solution just prior to centrifuging. This was followed by collection of the nanocapsule sediment, which was suspended in water with re-addition of glycerol $(20 \% v / v)$, which was frozen at $-75^{\circ} \mathrm{C}$ at a fairly slow freezing rate of $-1.25^{\circ} \mathrm{C} / \mathrm{min}$ (to minimize nanocapsule rupture) and maintained at this temperature for $48 \mathrm{~h}$ and, subsequently lyophilization at $-70{ }^{\circ} \mathrm{C}$ at 25 mtorr for a further $48 \mathrm{~h}$ (FreeZone $^{\circledR} 2.5$, Labconco $^{\circledR}$, Kansas City, MO, USA). It should be noted that the freezing temperature of the solution must be performed at, or below the glass transition temperature of the maximally cryo-concentrated solution for freezing to go to completion (thus at least at or below the $T_{\mathrm{g}}$ of PCL of $-60{ }^{\circ} \mathrm{C}$ ). The inclusion of Polysorbate 80 prevented the agglomeration of the nanocapsules on centrifugation and on freezing, while glycerol, a commonly-used freeze-drying excipient served as a cryoprotectant and tonicity adjustor, controlling the osmotic pressure.

\subsection{Analysis of Polymer Structure and Thermal Transition for Stability of Nanocapsule Constituents}

Owing to the fact that the melt dispersion technique is a process that uses relatively high temperatures, Fourier Transform Infrared (FTIR) spectroscopy, Differential Scanning Calorimetry (DSC) and Temperature-Modulated Differential Scanning Calorimetry (TMDSC) were performed to assess the possible presence of thermo-degradation.

\subsubsection{Fourier Transform Infrared Spectroscopy}

A Spectrum 100 FTIR Spectrometer (PerkinElmer Life And Analytical Sciences Inc., Shelton, CT USA) was used to detect the vibration characteristics of chemical functional groups in a sample, in response to infrared light interactions. The structures of PCL, CPZ and Polysorbate 80 were firstly assessed at room temperature. This was followed by heating of PCL, CPZ and Polsorbate 80 to $100{ }^{\circ} \mathrm{C}$, allowing it to cool and then assessing the structural backbones of these agents once again. Any obvious discrepancies between both sets of results would confirm possible changes to the chemical compounds and possible degradation. FTIR was also performed on CPZ-loaded PCL nanocapsule formulations to assess the potential for any variation in vibrational frequency caused as a result of nanocapsule formulation.

\subsubsection{Temperature-Modulated Differential Scanning Calorimetry}

DSC and TMDSC (DSC 1 STAR $^{\mathrm{e}}$ Mettler Toledo system, Uster, Zürich, Switzerland) were performed on native CPZ, PCL and the CPZ-loaded, PCL nanocapsules to further assess the thermal behavior of 
the constituents and thus pinpoint any thermo-degradation at the temperatures used in the melt dispersion technique. DSC and TMDSC were also performed to determine the possibility of any interactions that may have occurred during the melt dispersion technique. Calibration of temperature and enthalpy on the instrument were done using indium and zinc. The heating rate was set at $10^{\circ} \mathrm{C} \cdot \mathrm{min}^{-1}$ under a dry nitrogen atmosphere (Afrox, Germiston, Gauteng, South Africa) with a flow rate of $200 \mathrm{~mL} \cdot \mathrm{min}^{-1}$ acting as the purge gas in order to reduce oxidation. Samples sizes of 5-10 mg were accurately weighed into standard $40 \mu \mathrm{L}$ aluminum open pans and sealed hermetically. This was followed by pinholes being punched on the lids of the aluminum pans. Samples were then heated from 0 to $300{ }^{\circ} \mathrm{C}$ and held at $300{ }^{\circ} \mathrm{C}$ for $3 \mathrm{~min}$. This was done to evaporate any moisture in the sample and to eliminate any thermal history. The samples were then quenched from 300 to $0{ }^{\circ} \mathrm{C}$ at a rate of $20^{\circ} \mathrm{C} \cdot \mathrm{min}^{-1}$. The midpoint melting point $\left(T_{\mathrm{m}}\right)$ obtained from the melting point depression was determined according to the peaks generated on the experimental DSC curves on heating the samples from 0 to $300{ }^{\circ} \mathrm{C}$.

\subsection{Drug Entrapment and Yield of the PCL Nanocapsules}

The dry weight of the PCL nanocapsules was measured and compared to the dry weight of the initial formulation components. Chlorpromazine hydrochloride entrapment was determined by dissolving $100 \mathrm{mg}$ nanocapsules in $950 \mathrm{~mL}$ of Phosphate Buffered Saline (PBS), pH 7.4, $37^{\circ} \mathrm{C}$, to which $5 \mathrm{~mL}$ of acetone was added. Chlorpromazine hydrochloride content $(\mu \mathrm{g})$ was assessed in triplicate and determined by ultraviolet spectroscopy (Cecil CE 3021, Cecil Instruments Ltd., Milton, Cambridge, UK) at $319 \mathrm{~nm}$ against standard constructed curves. The entrapment efficiency was calculated as the actual percentage of drug entrapped compared to the original amount used in nanocapsule preparation.

\subsection{Size and Zeta-Potential Determination of the PCL Nanocapsules by Dynamic Light Scattering}

A Zetasizer NanoZS (Malvern Instruments Ltd., Malvern, Worcestershire, UK) incorporating dynamic light scattering techniques at $37^{\circ} \mathrm{C}$ at varying angles was used to determine the average sizes and size distribution of the nanocapsules produced, as well as their zeta potentials. A $1 \% w / v$ was appropriately diluted with de-ionized water, filtered using a $0.22 \mu \mathrm{m}$ filter (Millipore Co., Billerica, MA, USA) and placed into disposal cuvettes (size) or capillary cells (zeta potential) (Malvern Instruments Ltd., Malvern, Worcestershire, UK). The viscosity and refractive index of the continuous phase were set to those specific to de-ionized water. Measurements were taken in triplicate to validate the reproducibility of the experiment.

\subsection{Morphological Characterization of the PCL Nanocapsules}

Nanocapsule size and shape was explored in great detail utilizing cryo-Transmission Electron Microscopy (TEM) (JEOL 1200 EX, Tokyo, Japan, $120 \mathrm{keV}$ ). Samples were prepared by placing a dispersion of nanocapsules in ethanol on a copper grid with a perforated carbon film followed by evaporation and viewing at room temperature. 


\subsection{In Vitro Chlorpromazine Release Studies from the PCL Nanocapsules}

In vitro release studies were performed on the CPZ-loaded PCL nanocapsule formulations utilizing an orbital shaker bath (Labex, Stuart SBS40 ${ }^{\circledR}$, Gauteng, South Africa) set at $25 \mathrm{rpm}$. Due to the diminutive size of the nanocapsules and concerns of nanocapsule loss when sampling, one of the main methods reported for analysis of drug release from nanoparticulate systems was employed for in vitro drug release analysis, namely, the dialysis bag diffusion method, which has been employed in various modifications for evaluating drug release from nanoparticulate systems for neurological drug delivery $[10,12]$. We employed the most commonly cited dialysis bag (regular dialysis) method. Capsules equivalent to $5 \mathrm{mg} \mathrm{CPZ}$ were placed in dialysis tubing membranes and then immersed in $100 \mathrm{~mL}$ phosphate buffered saline (PBS) (pH 7.4, $37^{\circ} \mathrm{C}$ ) contained in $150 \mathrm{~mL}$ glass jars. At predetermined time intervals $3 \mathrm{~mL}$ samples of the release media were removed, filtered through a $0.22 \mu \mathrm{m}$ Cameo Acetate membrane filter (Millipore Co., Billerica, MA, USA) and analyzed by UV spectroscopy at a maximum wavelength of $319 \mathrm{~nm}$ for CPZ content analysis. Although the dialysis membrane should preclude their escape, the interference of nanocapsule remnants in filtered samples was accounted for, and found to be negligible, as ascertained by UV measurements at $319 \mathrm{~nm}$ on filtered samples obtained from drug-free nanocapsules. Further, preliminary experiments with $5 \mathrm{mg}$ pure $\mathrm{CPZ}$ from dialysis tubing showed no impedance of the drug release employing this approach. CPZ release was quantified using a linear standard curve $\left(R^{2}=0.99\right)$. To maintain sink conditions an equal volume of CPZ-free PBS was replaced into the release media. A model independent analysis, the time point approach, was used to analyze dissolution data to provide a more accurate analysis of CPZ release kinetics whereby mean dissolution time after 30 days (MDT30) was calculated using Equation (1):

$$
\mathrm{MDT}=\sum_{i=1}^{n} t_{i} \frac{M_{\mathrm{t}}}{M_{\infty}}
$$

where $M_{t}$ is the fraction of dose released in time $t_{i}=\left(t_{i}+t_{i}-1\right) / 2$ and $\mathrm{M}_{\infty}$ corresponds to the loading dose.

\subsection{Constraint Optimization of the Formulation Responses}

A model-independent approach (Minitab ${ }^{\circledR}$ V15, Minitab Inc., State College, PA, USA) was used to optimize the nanocapsules. Statistical optimization was therefore employed to ascertain the ideal polymeric and stabilizer combination with the desired physicochemical properties capable of attaining desirable nanocapsule sizes, zeta potentials and CPZ entrapment efficiencies.

\subsection{In Vitro Cytotoxicity Analysis in a Neuronal Cell Line}

The release of intracellular substances can be quantified by either initially labeling the cells exogenously or the intracellular substances itself may be physiological cytoplasmic constituents. Lactate dehydrogenase $(\mathrm{LDH})$ is a stable enzyme normally found in the cytosol of biological cells and is utilized in cytotoxicity assays as the quantification of LDH release from within the cell into the extracellular fluid (ECF) is proportional to cell death. A reduction in metabolic activity indicates incipient cell injury whereas LDH detection in the ECF represents more severe irreversible cell damage and implies death. 
The LDH assay assesses cell membrane integrity. Due to its advantages, the LDH assay method was employed in this study utilizing CytoTox $96^{\circledR}$ Non-Reactive Cytotoxic Assay. The effect of the nanocapsules and its componential elements were assayed to assess any cytotoxic effects on a neuronal cell line. PC12 neuronal cells were cultured in RPMI-1640 media comprising L-glutamine and sodium bicarbonate. The media was further enhanced with 5\% fetal bovine serum (FBS), 10\% horse serum (HS) and 1\% penicillin/streptomycin (Sigma-Aldrich; St. Louise, MO, USA). The FBS and HS were both heat inactivated. The cells were then maintained in a laboratory incubator (RS Biotech Galaxy, Irvine, UK) with a humidified atmosphere of $5 \%$ medical $\mathrm{CO}_{2}$ at $37^{\circ} \mathrm{C}$. Preparation of the media and inoculation of cells were conducted using strict aseptic technique in a laminar flow unit.

PC12 neuronal cells were exposed to the following compounds for $48 \mathrm{~h}$ :

a) Negative control (NC) - untreated cells

b) Positive control (PC) cells treated with $2 \%$ lysis buffer

c) CPZ-loaded nanocapsules $(20 \mathrm{mg})$

d) CPZ-free (non-drug loaded) nanocapsules (10 mg)

e) PCL (10mg)

The released LDH in the culture supernatants were determined using a CytoTox $96{ }^{\circledR}$ Non-Radioactive Cytotoxicity Assay. The released LDH was quantified utilizing a 30 minute enzymatic coupled test ultimately resulting in INT being converted to red formazan and catalyzed by LDH. Briefly, PC12 neuronal cells were added to the experimental microplate wells. These were treated with the compounds to be tested. This along with an untreated control was incubated for $48 \mathrm{~h}$ at $37^{\circ} \mathrm{C}$. After incubation, a lysis solution $(9 \% v / v)$ Triton $\left.{ }^{\circledR} \mathrm{X}-100\right)(10 \times)$, was added to all wells. The plate was then incubated at $37^{\circ} \mathrm{C}$ for $45 \mathrm{~min}$. LDH was quantified by firstly transferring $50 \mu \mathrm{L}$ of the supernatant of each well to another 96-well assay plate. The substrate mix was reconstituted using assay buffer and $50 \mu \mathrm{L}$ of this reconstituted substrate was added to each well. The assay plate was covered and incubated at room temperature, stored away from light, for $30 \mathrm{~min}$. This was followed by the addition of $50 \mu \mathrm{L}$ of stop solution to each well of the microplate. This was performed to stop the enzymatic reaction. Absorbance values at $490 \mathrm{~nm}$ were recorded utilizing a luminometer (Victor ${ }^{\mathrm{TM}} \mathrm{X} 3$, Perkin Elmer Inc., Waltham, MA, USA). The amount of LDH released and red color formed was directly proportional to the number of dead cells.

In order to relate the number of dead cells formed to the number of living cells and determine the cytotoxicity of the tested compounds, a target cell maximum LDH release control had to be conducted. A known quantity or density of PC12 neuronal cells were added to the culture medium in three wells of the microplate so that the final volume and concentration was the same as that utilized in the experimental assay above. In order to determine the known number of PC12 neuronal cells, the number of cells per $\mathrm{mL}$ was determined by utilizing a tryptan blue exclusion assay as well as a hemocytometer. The counting was based on the fact that viable cells will remain opaque and non-viable cells will stain blue and this would be quantified utilizing a hemocytometer and light microscope. Briefly, $200 \mu \mathrm{L}$ of the cell suspension was diluted with $300 \mu \mathrm{L}$ PBS and added to $500 \mu \mathrm{L}$ tryptan blue thereby creating a dilution factor of 5. The dilution factor was relatively high so as to easily identify individualized cells. The suspension was mixed thoroughly and allowed to stand for $10 \mathrm{~min}$. A Pasteur pipette was used to transfer a miniscule quantity of the suspension to the chamber of 
the hemocytometer. The cells in the $1 \mathrm{~mm}$ square and four corners were counted. Each square of the hemocytometer is equal to a volume of $0.1 \mathrm{~mm}^{3} / 10^{-4} \mathrm{~cm}^{3}$ and since $1 \mathrm{~cm}^{3}=1 \mathrm{~mL}$, the cell count per $\mathrm{mL}$ could be calculate utilizing Equation (2). Only cell suspensions with a viability in excess of 95\% were used in the assay:

$$
\mathrm{PC} 12 \text { neuronal cells } / \mathrm{mL}=\text { Average count per square } \times \text { dilution factor } \times 10^{4}
$$

Once the cells were counted and pipetted onto a microplate, $10 \mu \mathrm{L}$ of lysis solution per $100 \mu \mathrm{L}$ of culture medium was added and the cells together with the lysis solution were incubated at $37{ }^{\circ} \mathrm{C}$ for 45 min. This resulted in complete lysis and death of the PC12 neuronal cells. The supernatants were harvested as described above and the ultraviolet reading at $490 \mathrm{~nm}$ for complete cell destruction was computed. Volume correction as well as culture medium controls was performed to cater for the volume changes by addition of lysis buffer as well as correcting LDH activity contributed by serum in culture medium and the phenol red in the culture medium respectively. Furthermore, a target-cell spontaneous LDH release control was also performed to correct spontaneous LDH release from cells. The cytotoxicity of the compounds towards the PC12 neuronal cells were calculated using Equation (3):

$$
\% \text { Cytotoxicity }=\frac{\text { Experimental LDH Release }}{\text { Maximum LDH Release }} \times 100
$$

The statistical significance of the cytotoxic effects of the various compounds was evaluated wherein a probability limit of $p<0.05$ was considered to be significant.

\subsection{Assimilation of the Optimized Nanocapsules into a Composite Polymeric Membrane for Implantation}

The assembly of a nanocapsule-loaded polymeric membrane (NCPM) was carried out by incorporating and evenly dispersing the optimized CPZ-loaded, PCL-based nanoparticle formulation into a polyamide (PA) 6,10-ethylcellulose (EC) composite membrane, derived from a polymeric slurry, with subsequent molding to obtain a composite membrane. The PA 6,10 utilized in this study is a novel polymer synthesized by Kolowale and co-workers [20]. For preparation of the membrane slurry, PA 6,10 was dissolved in $2 \mathrm{~mL}$ Formic Acid (FA). The solution was heated to $60{ }^{\circ} \mathrm{C}$ to aid the complete solubilization of the PA 6,10 in the FA. A separate solution was formulated and comprised of EC dissolve in $1 \mathrm{~mL}$ of acetone $(\mathrm{ACN})$. Once the PA 6,10 and the EC were completely solubilized in their respective solvents, the PA 6,10-FA solution was the gradually added to the EC-ACN solution with stirring at $500 \mathrm{rpm}$. This led to the formation of a white, murky slurry of consistent texture. The slurry was then removed and added to a dialysis tubing membrane which was then suspended in $5 \mathrm{~L}$ of de-ionized water. As water permeated through the dialysis tubing and came into contact with the slurry, it led to the formation of a white precipitate. Once all FA was removed, half of the PA 6,10-EC slurry post dialysis was placed in the liquid-paraffin lubricated molds. This was followed by the addition of $500 \mathrm{mg}$ of optimized nanoparticles and the further addition of the remainder of the slurry. The molds were then placed under a fume hood and air-dried for $24 \mathrm{~h}$. The final NCPM was sterilized via gamma irradiation at $20 \mathrm{kGy}$. 


\subsection{In Vivo Analysis of the Nanocapsule-Loaded Polymeric Membrane in a Rabbit Model}

\subsubsection{Implantation of the Nanocapsule-Loaded Polymeric Membrane into a Rabbit Model}

New Zealand White Rabbits were utilized in this study and were obtained as per the protocols of the Central Animal Services at the University of the Witwatersrand. Ethics clearance from the Animal Ethics Screening Committee (AESC) of the University of the Witwatersrand was obtained for this study (No. 2011/51/04 Eight rabbits weighing 4.5-5 kg were housed in single cages, i.e., one rabbit per cage and were provided with a standard rabbit diet and water ad libitum under a controlled temperature $\left(20-24{ }^{\circ} \mathrm{C}\right)$ and a $12 \mathrm{~h} \mathrm{light/dark} \mathrm{cycle.}$

In vivo assessment was conducted on 6 rabbits. Two rabbits were assigned to 3 sub-groups where the first sub-group was implanted with a drug-loaded NCPM (Group 1), the second with a non drug-loaded NCPM (for baseline comparative purposes) (Group 2) and the third with an intramuscular parenteral dose of commercially available CPZ (Chlorpromazine HCl-Fresenius ${ }^{\circledR} 25 \mathrm{mg} / \mathrm{mL}$ ) (Group 3). The dose of the intramuscular CPZ injection was $1 \mathrm{mg} / \mathrm{kg}$ of body weight. This dose was attained by the veterinary formulary of the University of Minesota. One rabbit from each group was euthanized approximately halfway through the study (Day 14) and was subjected to histolomorphological toxicity studies. The remaining rabbits were euthanized at the end of the study (Day 30) and underwent further histolomorphological toxicity studies.

The implantation was conducted on live, healthy, rabbits. Rabbits were initially given half the recommended dose of midazolam to as a sedating agent followed by anaesthesia with a mixture of Ketamine $(50 \mathrm{mg} / \mathrm{kg})$ and Xylazine $(5 \mathrm{mg} / \mathrm{kg})$. The implantation procedure was adapted from Brem and co-workers [21] and is briefly outlined; the heads of the animals were shaven and appropriately prepared. A midline incision was made and this was followed by the removal of subcutaneous tissue and periosteum. A unilateral burrhole of approximately $5 \mathrm{~mm}$ was made using a dental drill. The burrhole was located anterior to the coronal suture and lateral to the saggital suture. The dura mater and cortex were pierced with minimal resultant bleeding. The burrhole was flushed with saline and the implant was placed intraparenchymally. The scalp insertion was then appropriately closed with a single layer of non-absorbable suture.

\subsubsection{Histomorphological Evaluation of the Brain Post-Implantation}

Histomorphological analysis was conducted to assess the biocompatibility of the NCPM and its immune response in terms of its inflammation response, whether or not it is toxic or causes excessive bleeding. Rabbits were euthanized midway through the study (Day 14) and at the end of the study (Day 30) with sodium pentobarbitone and decapitated. The entire brain was removed and fixed in $10 \% v / v$ neutral buffered formalin solution. Following the normal fixation period, the brain specimens were cut by cross section in the anterior third region where the NCPM was implanted or where areas of lysis could be identified. Additional sections in the mid-brain and cerebellum were also examined to assess any effects of nanocapsules, drug or the NCPM that may have translocated to these areas as a result of biodegradation. These selected blocks were then processed in an automated histological processor overnight. After complete tissue processing, wax blocks were prepared and histological sections of $6 \mu \mathrm{m}$ were cut on a 
microtome. The slides produced were stained with haematoxylin and eosin ( $\mathrm{H} \& \mathrm{E})$ in an automated stainer prior to mounting for histomorphological evaluation.

\subsubsection{Cerebrospinal Fluid Sampling}

Cerebrospinal fluid (CSF) samples $(500 \mu \mathrm{L})$ were be drawn by cisternal puncture by a method. Briefly, the rabbits were anesthetized and placed in lateral recumbency, with the head flexed at $90^{\circ}$ to the neck. The fur was then shaved on the dorsal head and neck and the site was appropriately disinfected. A 23 gauge hypodermic needle was inserted into the dorsal midline between the external occipital protuberance and the craniodorsal tip of the dorsal spine of the $\mathrm{C} 2$ vertebra, cranial to the cranial wings of the $\mathrm{C} 1$ vertebra. The needle then gently entered the subarachnoid space and CSF was passively withdrawn into $1 \mathrm{~mL}$ syringes and stored in Eppendorf tubes at $-80^{\circ} \mathrm{C}$ till further analysis. Any contaminated CSF samples were centrifuged at 1300 relative centrifugal force (RCF) and the clear supernatant was collected and stored immediately at $-80{ }^{\circ} \mathrm{C}$ in a laboratory freezer. Blank CSF for base-line data was withdrawn from the rabbit 1 week prior to implantation with the membrane.

\subsubsection{Blood Sampling}

Due to the fact that the link between CSF and the venous blood flow are the arachnoid granulations, substances within the CSF can enter the blood stream via the arachnoid granulations [22]. Blood samples were therefore taken to assess the potential levels of $\mathrm{CPZ}$ that may have enter the systemic circulation due to the minute size of the nanocapsules and its potential ability to pass through the arachnoid granulations. Blood samples were withdrawn at specific time intervals through the marginal ear vein of the rabbit on days $0,3,7,14,21$ and 30. An intravenous catheter (22 gauge) was used to draw approximately $1 \%$ of the animal's body weight, i.e., $3 \mathrm{~mL}$ of blood from a $3 \mathrm{~kg}$ rabbit and $4.5 \mathrm{~mL}$ of blood from a $4.5 \mathrm{~kg}$ rabbit. These guidelines were attained from "The Guidelines for Collection of Blood from Experimental Animals", Research Animal Resources, University of Minnesota. Blood sampling was conducted on anesthetized rabbits. Blank blood for base-line data was withdrawn from the rabbit 1 week prior to implantation with the membrane. The blood samples were collected into heparinised vacutainer tubes and gently mixed to prevent clotting. The samples were centrifuged at $1300 \mathrm{RCF}$ soon after. The supernatant, containing the plasma, was carefully aspirated and transferred into a clean appropriate collection tube and frozen at $-80^{\circ} \mathrm{C}$ immediately till further analysis.

\subsubsection{Analysis of CSF and Blood Samples}

UPLC was performed utilizing a Waters Acquity ${ }^{\circledR}$ UPLC system (Waters Corp., Milford, MA, USA). The system contains a binary solvent manager, sample manager and a photodiode array (PDA) detector, all connected to a Waters Empower 2 data station. An Acquity UPLC Ethylene Bridged Hybrid (BEH) C18 column $(50 \mathrm{~mm} \times 2.1 \mathrm{~mm}$, i.d., $1.7 \mu \mathrm{m}$ particle size, Waters $)$ was used at $40{ }^{\circ} \mathrm{C}$ to achieve separation. The machine was primed with the appropriate solvents mentioned above for 10 cycles each of $5 \mathrm{~min}$. The method for separation, identification and quantification was isocratic whereby the mobile phase comprised ammonium acetate buffer and acetonitrile (AA-ACN 65\%-35\% v/v), the flow rate through the column was $0.50 \mathrm{~mL} / \mathrm{min}$, the injection volume was $2 \mu \mathrm{L}$ and the run time was 3 min. Between 
injections, the machine was allowed to equilibrate for $3 \mathrm{~min}$ so as to attain a constant pressure and more reliable results. Upon completion of analysis, ACN was gradually injected into the system to remove any residue until the entire column was immersed in $100 \% \mathrm{ACN}$. Isoniazid (INH) was employed as the internal standard (IS). The PDA detector was set at $254 \mathrm{~nm}$ for $\mathrm{CPZ}$ and INH detection. Standards were prepared and analyzed (ensuring precision and accuracy) for calibration curve construction.

\subsubsection{Plasma and CSF Extraction of CPZ}

An uncomplicated and effective liquid-liquid plasma extraction procedure was developed and applied to $\mathrm{CPZ}$ in blood samples. Aliquots of plasma $(500 \mu \mathrm{L})$ of sample were transferred into polypropylene tubes followed by the addition of $1 \mathrm{~mL}$ of $\mathrm{ACN}$ in order to precipitate the plasma proteins. The samples were subsequently vortexed for 1 min followed by the addition of $500 \mu \mathrm{L}$ of ACN. The mixture was vortexed again for $1 \mathrm{~min}$ and was then centrifuged at 1300 RCF (Nison Instrument Limited, Shanghai, China) for $5 \mathrm{~min}$. The supernatant was removed and filtered through $0.22 \mu \mathrm{m}$ Cameo Acetate membrane filters. The filtrate was then spiked with a known amount of the IS, INH. The final solution was transferred into Waters ${ }^{\circledR}$ certified UPLC vials for analysis. Due to the fact that CFS does normally contain proteins and that $\mathrm{CPZ}$ is highly protein bound, the same extraction procedure was applied to CSF samples.

\section{Results and Discussion}

The melt dispersion technique is a non-arduous technique that does not require the use of organic solvents as do most conventional methods for conventional nanocapsule synthesis. This is highly beneficial considering the toxicity associated with organic solvents and the cost implications thereof. The thermoplastic nature of PCL has proved to be of great benefit in that PCL can easily be manipulated once passed its melting point and exposed to high shearing force. Nanocapsule collection post curing is one of the major difficulties associated with the melt dispersion technique. The high viscosity of glycerol makes separation and centrifuging of the capsules complex as capsules tend to be dispersed in the supernatant as opposed to sedimenting. This was overcome by the addition of de-ionized water to the nanocapsule solution post curing. The miscibility of water with glycerol allowed for a decrease in viscosity of the solution which made it more favorable to centrifuging and the nanocapsule sedimenting thereby allowing for collection, freezing and lyophilizing, forming a free-flowing powder (Figure 2). The addition of water could however impact parameters such as drug-loading and diffusion of the highly water-soluble CPZ out of the nanocapsules.

\subsection{Assessment of Polymeric Structure for Thermo-Degradation of Nanocapsule Constituents}

FTIR was performed on PCL, CPZ and Polysorbate 80 at room temperature as well as samples of PCL, CPZ and Polysorbate 80 which were heated to $100^{\circ} \mathrm{C}$ and then allowed cool (Figure 3). Spectra obtained for room temperature samples as well as samples that were heated to $100{ }^{\circ} \mathrm{C}$ and cooled were super-imposable. This thereby confirmed that the temperatures used for the melt dispersion technique did not degrade each of the components utilized in the melt dispersion technique. 

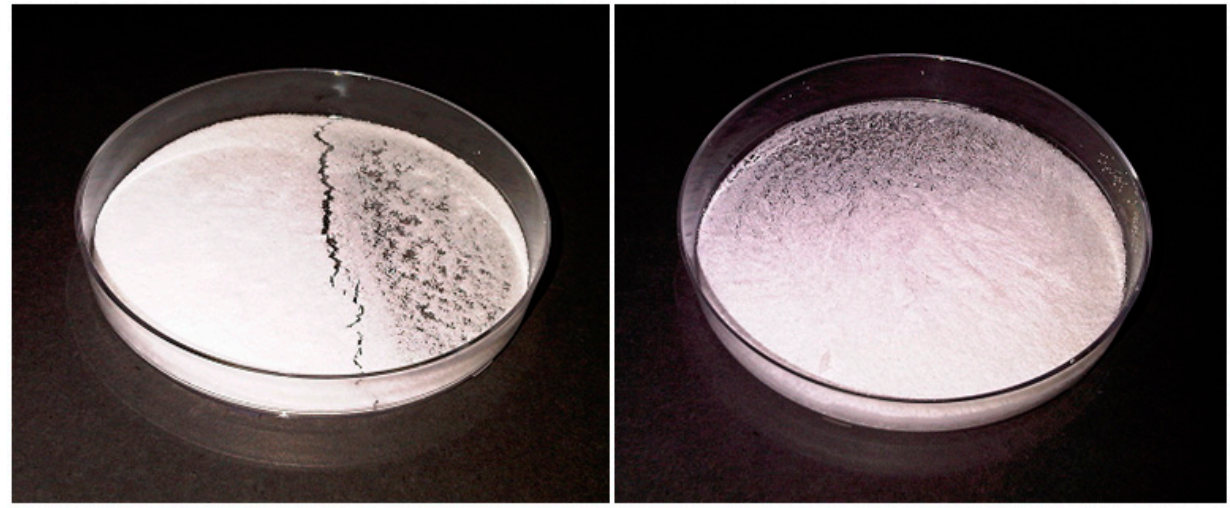

Figure 2. Digital images of PCL nanocapsule formulations post lyophilization.
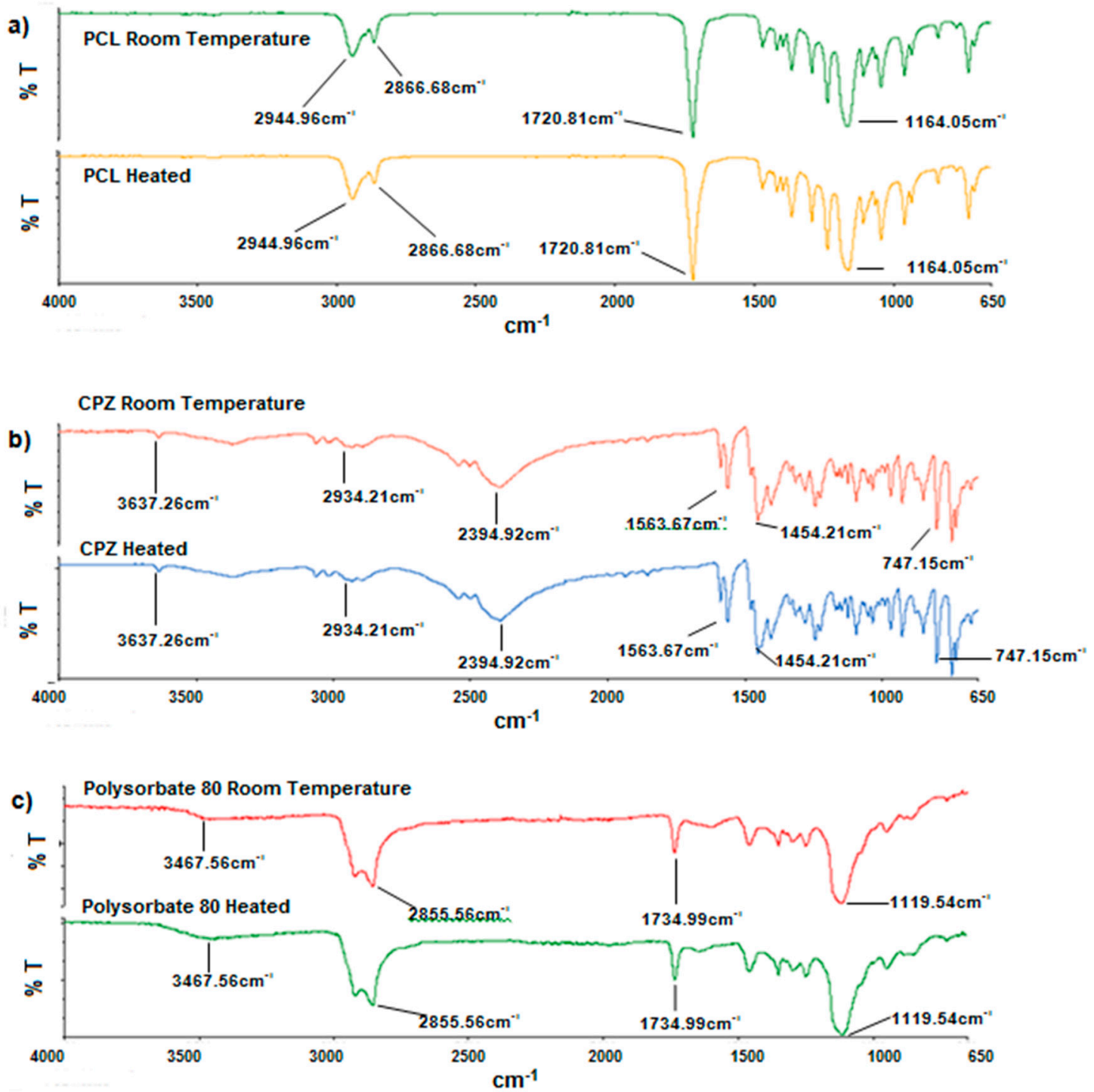

Figure 3. FTIR spectra of (a) PCL (room temperature and heated); (b) CPZ (room temperature and heated, and (c) Polysorbate 80 (room temperature and heated). 
The characteristic peaks of PCL $\left(2944.96 \mathrm{~cm}^{-1}, 2866.68 \mathrm{~cm}^{-1}, 1720.81 \mathrm{~cm}^{-1}, 850-1480 \mathrm{~cm}^{-1}\right)$ all appear in both PCL at room temperature and PCL heated to $100{ }^{\circ} \mathrm{C}$ then cooled to room temperature. $2944.96 \mathrm{~cm}^{-1}$ is indicative of asymmetric $\mathrm{CH}_{2}$ stretching whereas the peak at $2866.68 \mathrm{~cm}^{-1}$ is indicative of symmetrical $\mathrm{CH}_{2}$ stretching. The strong band at $1720.81 \mathrm{~cm}^{-1}$ is a result of the carbonyl stretching vibration [23,24]. The characteristic peaks of CPZ $\left(2934.21 \mathrm{~cm}^{-1}, 2394.92 \mathrm{~cm}^{-1}, 1563.67 \mathrm{~cm}^{-1}, 1458.12 \mathrm{~cm}^{-1}\right.$, $730-850 \mathrm{~cm}^{-1}$ ) all appear in both $\mathrm{CPZ}$ at room temperature and $\mathrm{CPZ}$ heated to $100{ }^{\circ} \mathrm{C}$ then cooled to room temperature and are indicative of aliphatic $\mathrm{C}-\mathrm{H}$ stretching $\left(2934.21 \mathrm{~cm}^{-1}\right), \mathrm{H}_{3} \mathrm{C}-{ }^{+} \mathrm{NH}$ stretching and combination modes $\left(2394.92 \mathrm{~cm}^{-1}\right)$, Phenyl ring vibrational modes $\left(1563.67 \mathrm{~cm}^{-1}\right), \mathrm{C}-\mathrm{H}$ deformational modes $\left(1458.12 \mathrm{~cm}^{-1}\right)$ and aromatic $\mathrm{C}-\mathrm{H}$ bending modes $\left(730-850 \mathrm{~cm}^{-1}\right)$.

The characteristic peaks of Polysorbate $80\left(3467.56 \mathrm{~cm}^{-1}, 2855.56 \mathrm{~cm}^{-1}, 1734.99 \mathrm{~cm}^{-1}\right.$ and $1119.54 \mathrm{~cm}^{-1}$ ) appear on both Polysorbate 80 at room temperature as well as heated Polysorbate 80 and correspond to the stretching vibrations of $\mathrm{O}-\mathrm{H}, \mathrm{C}=\mathrm{O}, \mathrm{C}-\mathrm{O}-\mathrm{C}$ bonds respectively [25]. The mere fact that all major peaks of each of PCL, CPZ and Polysorbate 80 remained intact and were reproducible post heating to $100{ }^{\circ} \mathrm{C}$ and cooling confirms that the chemical backbones of each compound exhibited no thermodegradation and that the melt dispersion technique was a viable nanocapsule synthesis technique.

DSC thermograms of CPZ, PCL and the CPZ-loaded PCL nanocapsules revealed strong endothermic peaks at $198.20^{\circ} \mathrm{C}, 66.19^{\circ} \mathrm{C}$ and $61.99{ }^{\circ} \mathrm{C}$ respectively and corresponds to the melting transition $\left(T_{\mathrm{m}}\right)$ of these compounds (Figure 4). The continuous baseline on either side of the melting points of CPZ and PCL indicate that these compounds melt without degradation. With regards to CPZ, a decline in the baseline is noted from $230{ }^{\circ} \mathrm{C}$ onwards. This could be a result of degradation. Despite this, the baseline covers and goes beyond temperatures used in the melt dispersion technique thus confirming that there is no thermodegradation when utilizing the melt dispersion technique. The $T_{\mathrm{m}}$ of the CPZ-loaded PCL nanocapsules exhibited a decrease from 66.19 to $61.99^{\circ} \mathrm{C}$ when compared to pure PCL. Furthermore, a pronounced endotherm at $39.54{ }^{\circ} \mathrm{C}$ was observed and overlaps with the melting endotherm of the nanocapsules. TMDSC (Figure 4d) revealed that the peak at $39.54{ }^{\circ} \mathrm{C}$ does not occur and is an unexpected transition in the DSC thermogram. In addition, transitions pertaining to the Non-reversible heat flow curve and the Total heat flow curve occur at $58.29{ }^{\circ} \mathrm{C}$. Transitions on these curves are indicative of crystallization or melting. The fact that the transition is endothermic confirms melting is occurring.

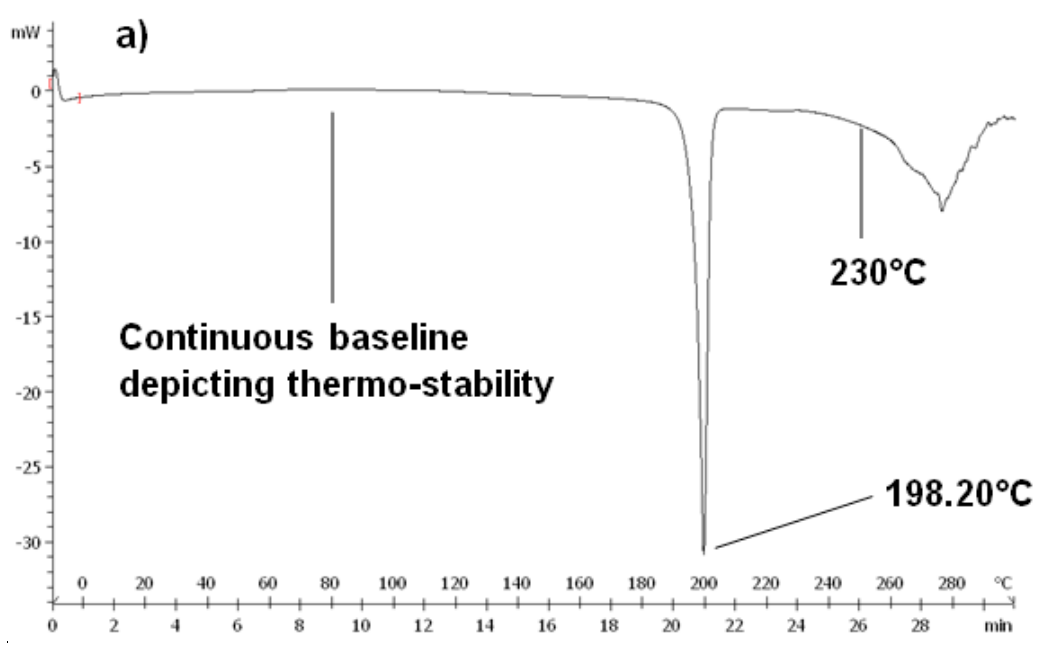

Figure 4. Cont. 


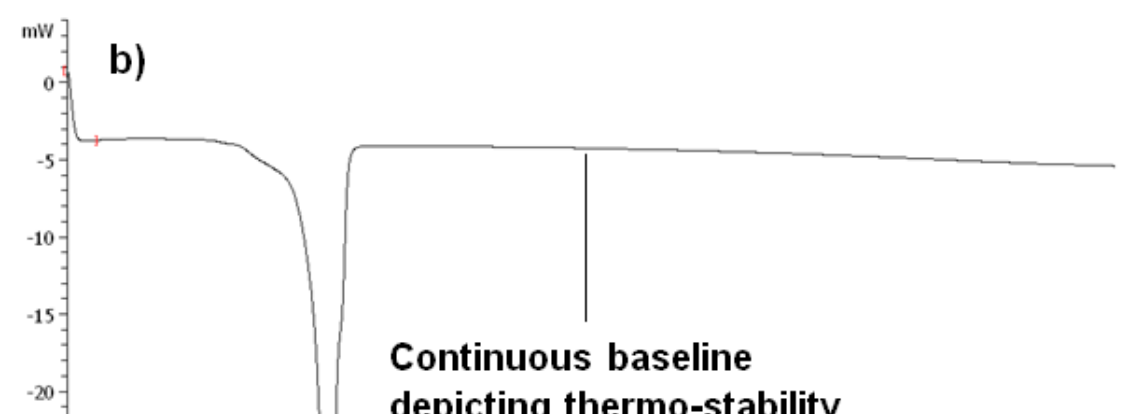

\section{depicting thermo-stability}

$66.19^{\circ} \mathrm{C}$
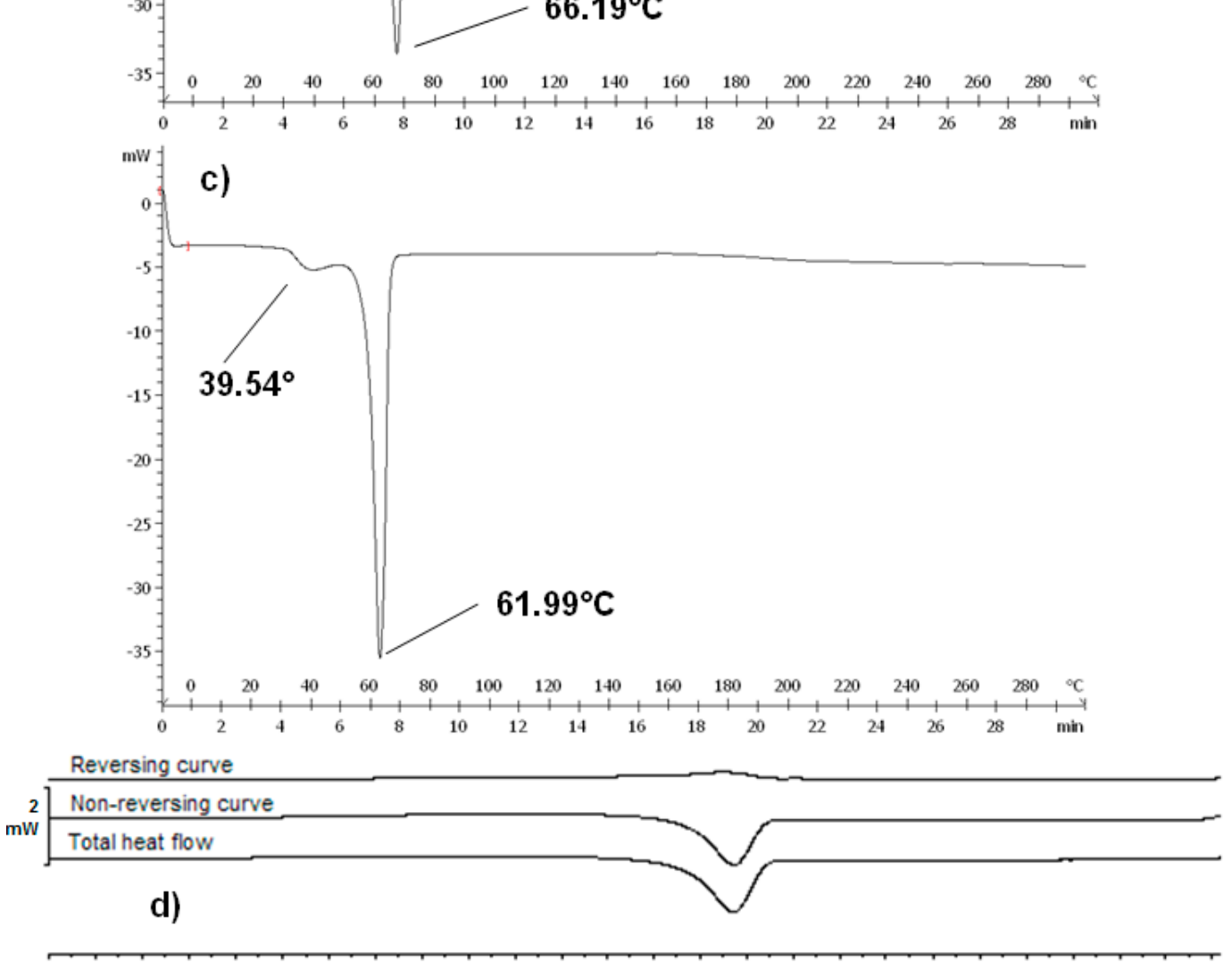

Figure 4. DSC of (a) CPZ (b) PCL and (c) CPZ-loaded PCL based nanocapsule formulation and TMDSC profile of d) CPZ-loaded PCL based nanocapsule formulation between 30 and $80{ }^{\circ} \mathrm{C}$.

The moderate decrease in melting point of the drug-loaded PCL could possibly be as a result of the incorporation of $\mathrm{CPZ}$ which may in fact restrict the periodic arrangement of PCL chains into its lattice thereby causing the crystalline structure of PCL to be less perfect in the nanocapsule form as opposed to pure PCL [26].

\subsection{Drug Entrapment and Yield of PCL Nanocapsules}

Nanocapsule yield from the 13 formulations ranged from $89.58 \%$ to $99.54 \%$ with an average of $94.74 \%$ and is illustrated in Figure 5. The yield of non-drug loaded nanocapsules was $99.23 \% \pm 2.82 \%$. High yield values are due to the relatively high amount of PCL used in each formulation. 


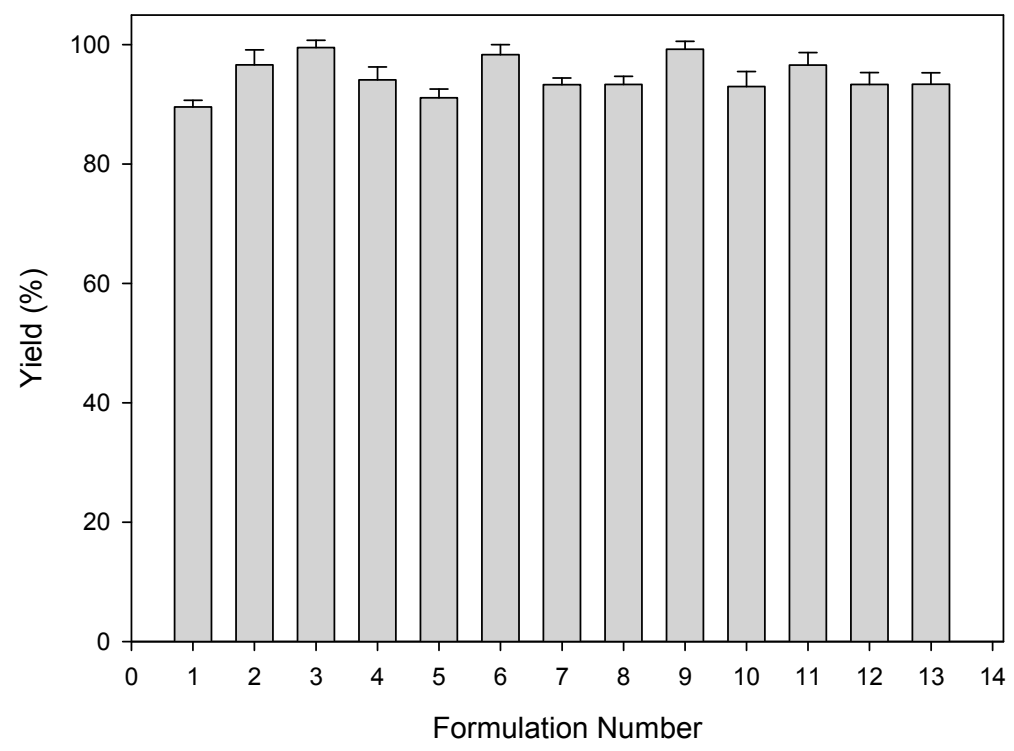

Figure 5. Nanocapsule yield of each formulation as per statistically generated design template ( $\mathrm{SD} \leq 2.52$ in all cases, $N=3$ ).

The amount of CPZ entrapped within the PCL nanocapsules ranged from 1933.51 to $8230 \mu \mathrm{g}$ (SD $\leq 60.34, n=3$ ) with entrapment efficiencies of $9.67 \%-41.15 \%$. Figure 6 provides the amount of CPZ entrapped within the PCL nanocapsules for each formulation. The relatively low CPZ entrapment values bear testament to the fact that $\mathrm{CPZ}$ is highly hydrophilic and PCL itself is highly hydrophobic hence both entities are less chemotactic towards each other. Furthermore, low CPZ entrapment values could also be due to the addition of water to the formed nanocapsule solution post curing to aid in decreasing the viscosity of the solution, centrifuging and collection of nanocapsules. Further leaching of $\mathrm{CPZ}$ out of the nanocapsules could also be a result of washing out the capsules during the collection process of the nanocapsule synthesis. The highly hydrophilic CPZ has a great affinity towards the aqueous phase invariably resulting in massive amount of drug loss. Nanocapsules in general, due to their diminutive sizes and large surface areas allow for very limited amounts of drug to be entrapped [27].

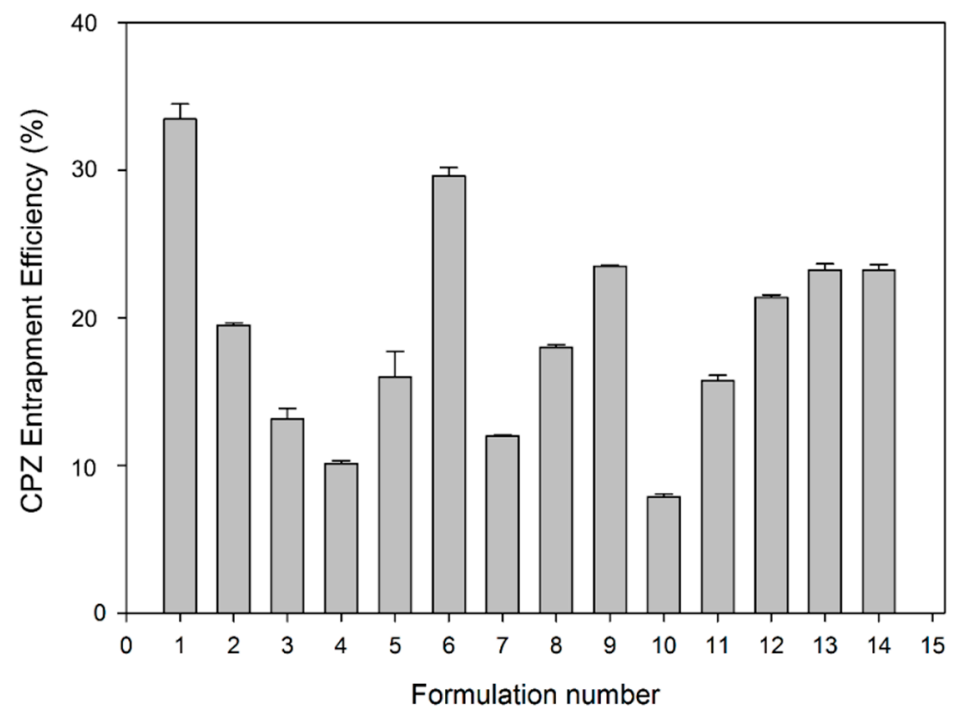

Figure 6. Percentage drug entrapment of CPZ within PCL nanocapsules as per the statistical experimental design template generated $(N=3)$. 


\subsection{Assessment of PCL Nanocapsules Size and Zeta-Potential}

The $z$-average size of CPZ-loaded, PCL based nanocapsules ranged from 132.7 to $566.6 \mathrm{~nm}$ as per experimental design template and is depicted in Figure 7a. Figure 8 shows the size and zeta potential profiles for Formulation 8, which possessed a good average size and favourable zeta potential. It can be seen that the sizes of the nanocapsules are significantly smaller, i.e., $63.46 \mathrm{~nm}$ for non-drug loaded particles as compared to $132.70 \mathrm{~nm}$ of the drug load-loaded nanocapsules. This is a typical result as the addition of drug to the particle would generally result in an increase in particle size. In general, increasing amounts of PCL above $3000 \mathrm{mg}$ and concentration of Polysorbate 80 above $5 \%$ resulted in larger capsules which are typical findings. The nanocapsule sizes achieved were desirable and in the range for nano-pharmaceutical applications. Zeta potential values ranged from -15.1 to $-28.8 \mathrm{mV}$ and are depicted in Figure $7 \mathrm{~b}$. These results represent nanocapsules that are incipiently to moderately stable. Increased concentrations of Polysorbate 80 resulted in capsules with high absolute zeta potential values whereas a decrease in Polysorbate 80 concentration below 2\% resulted in lower absolute zeta potentials and unstable capsules that tend to aggregate. Furthermore, the absolute zeta potential of non-drug loaded particles $(-10.80 \mathrm{mV})$ was lower than that of drug-loaded nanocapsules $(-28.80 \mathrm{mV})$ and were less stable, possibly indicating stabilization of the nanocapsules by the drug. The stability of the nanocapsules is maintained by the steric interactions and weak electrostatic repulsive forces of adsorbed Polysorbate 80 on the surface of the nanocapsules [28,29].
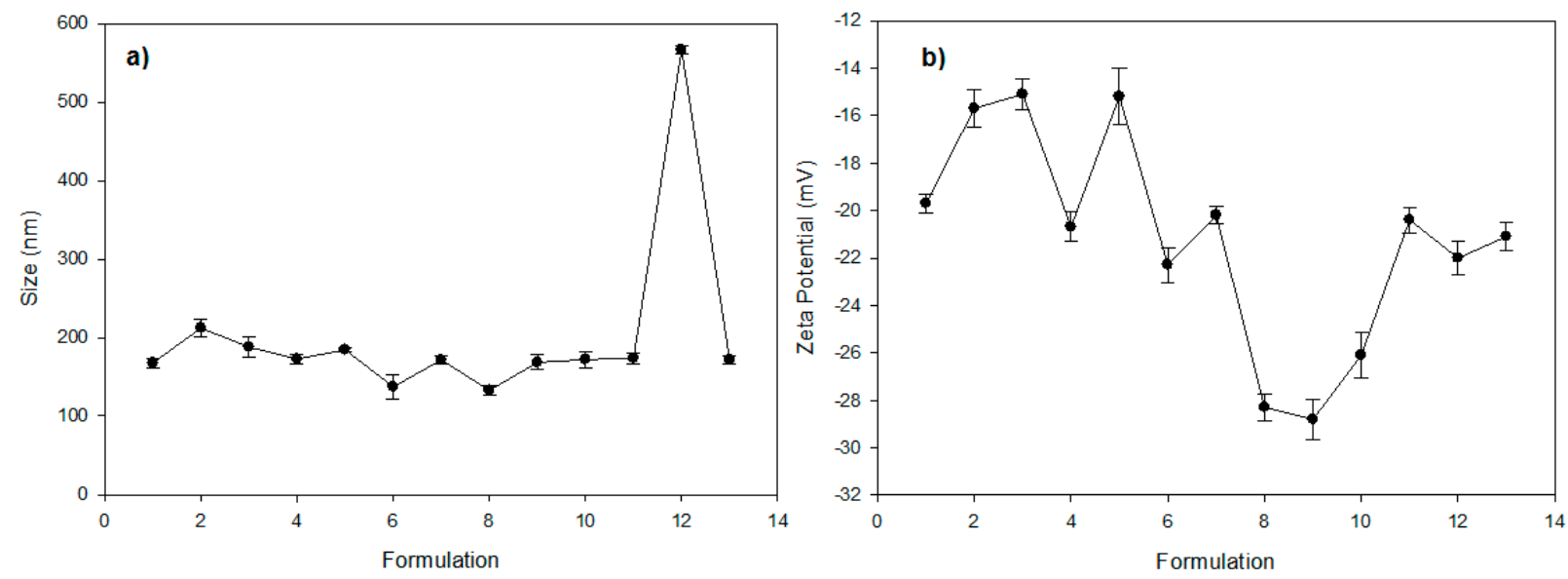

Figure 7. Graphical representation of (a) sizes achieved and (b) zeta potentials achieved for CPZ-loaded PCL based nanocapsules as per statistical design.

a)

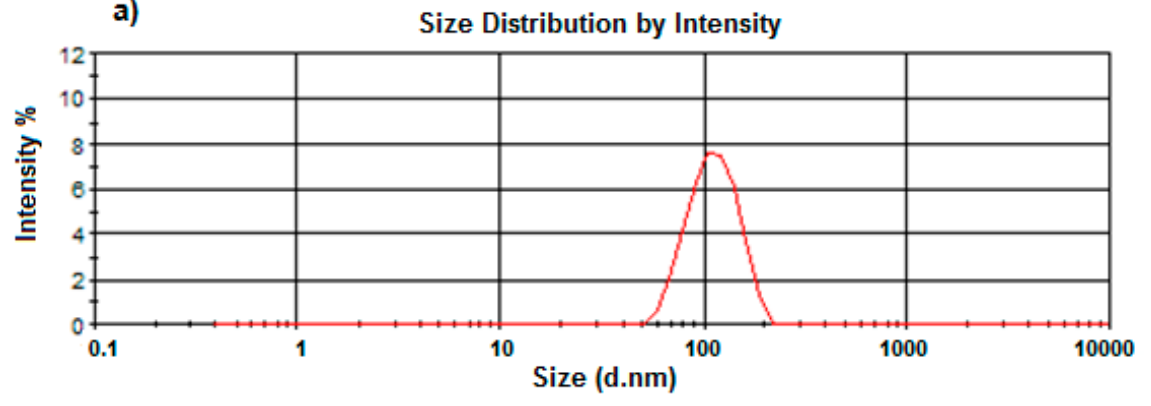

Figure 8. Cont. 
b)

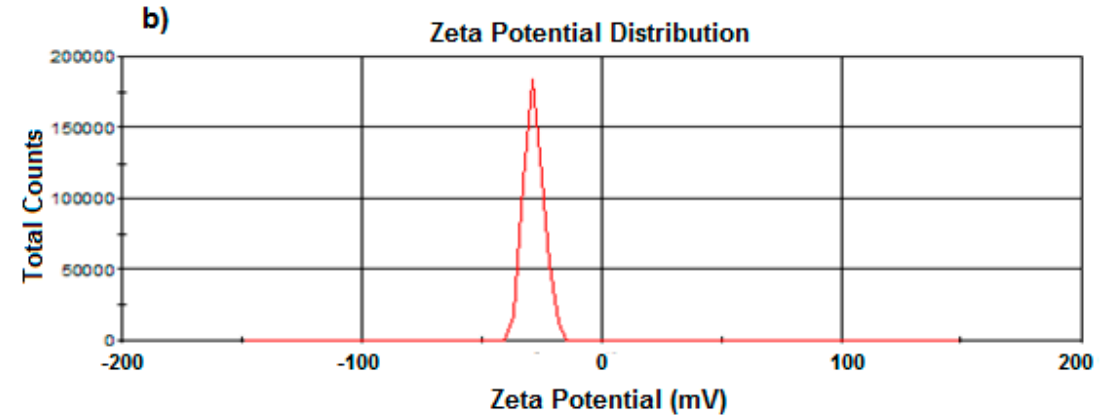

c)

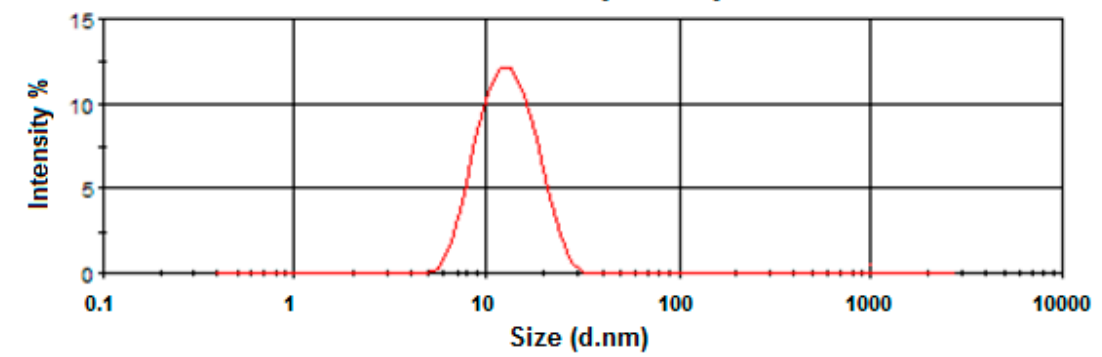

d)

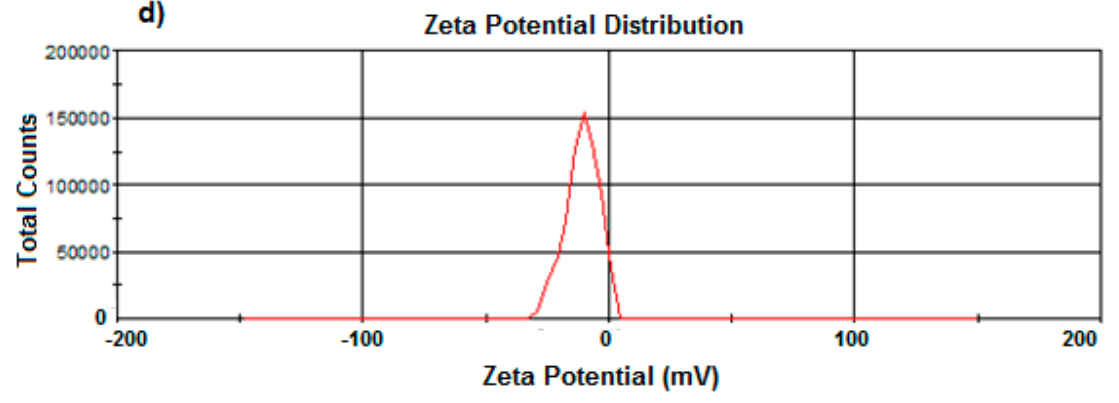

Figure 8. Distribution profiles of (a) size and (b) zeta potential of drug-loaded nanocapsules of Formulation 8 of the experimental design. Distribution profiles of (c) size and (d) zeta potential of non-drug loaded nanocapsules of Formulation 8 are also depicted.

\subsection{Morphology of the PCL Nanocapsules}

TEM revealed spherical capsules which were of consistent size. CPZ-free capsules appeared denser in structure whereas CPZ-loaded capsules appeared to be opaque. Both drug-loaded and non-drug-loaded capsules exhibited a high degree of flocculation which is consistent with zeta potential results, thereby displaying moderate and incipient stability from weak steric forces instead of a highly stable, de-flocculated suspension. Despite this, capsules were well individualized due to incorporation of Polysorbate (Figure 9).

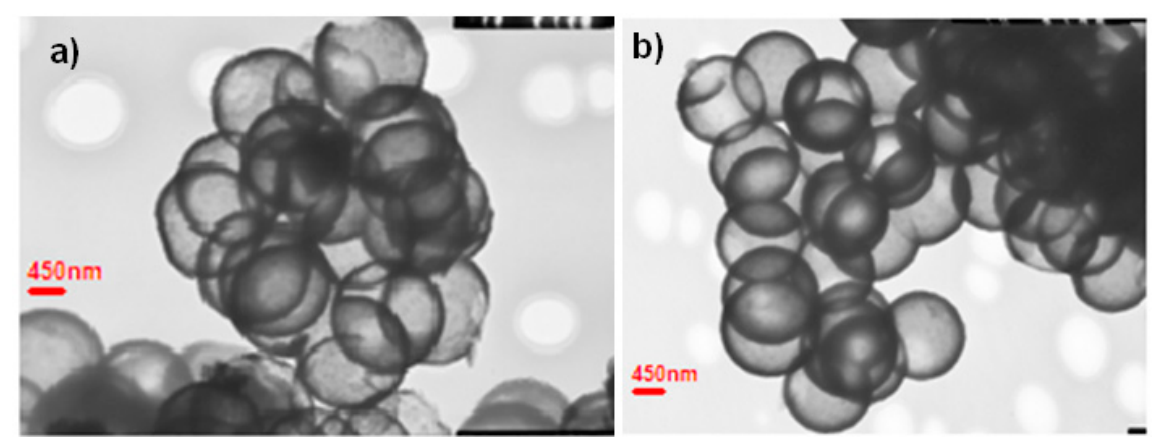

Figure 9. Cont. 

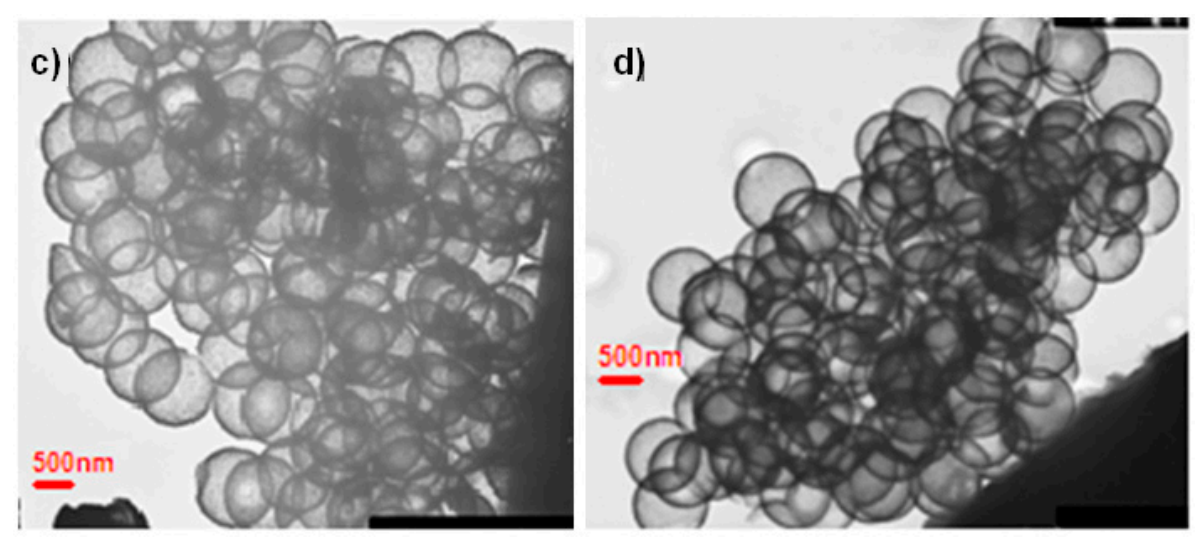

Figure 9. TEM of $(\mathbf{a}, \mathbf{b})$ Non-drug loaded, PCL based nanocapsules and (c,d) CPZ-loaded PCL based nanocapsules (Magnification 50,000× (a and b); 40,000× (c and d)).

\subsection{In Vitro Drug Release Studies}

All formulations of the experimental design exhibited biphasic CPZ release characteristics with an initial burst release followed by controlled pseudo-steady release over a period of 30 days and is depicted in Figure 10. The initial burst release is characteristic of PCL nano and microcapsules and is mainly due to weakly bound or adsorbed CPZ on the surface of the PCL nanocapsule compounded by the highly hydrophilic nature of CPZ which generally tends to dissolute in aqueous media (PBS) rapidly. The burst release could be as a result of CPZ diffusion through the superficial layers of the PCL nanocapsule whereas the controlled, steady release kinetics could be a result of CPZ diffusing from the inner PCL matrix as a result of polymer degradation [30]. Other possible reasons for the initial burst is attributed to nanocapsule sizes, drug-loading, low molecular weight of the drug and drying conditions [15,31], and is elaborated on below.

Larger capsules generally have a smaller initial burst release and a longer sustained release than smaller capsules. This is confirmed by comparing the release kinetics of Formulation 6 to the release kinetics of Formulation 12 in the experimental design. Formulation 6 had a $z$-average nanocapsule size of $137.2 \mathrm{~nm}$ and exhibited an initial burst release of $58.3 \%$ of the entrapped CPZ. Formulation 12 had a $z$-average nanocapsule size of $566.6 \mathrm{~nm}$ and displayed a burst release of $19 \%$ of entrapped CPZ. Furthermore at day 30, Formulation 6 had release $69.5 \%$ of entrapped CPZ whereas Formulation 12 had released $22.4 \%$. In addition, greater nanocapsule drug-loading results in greater burst release effects [15]. This again is confirmed by comparing Formulations 6 and Formulation 12 with a CPZ entrapment of 3286.56 and $1896.04 \mu \mathrm{g}$ respectively. CPZ-loading of PCL nanocapsules in general was extremely low, hence the burst release effects of the majority of the formulations in the experimental design were relatively low. Furthermore, all formulations of the experimental design displayed controlled release of CPZ after the initial phase with drug release ranging from $22.4 \%$ to $69.5 \%$ after 30 days. This highlights the potential of the synthesized formulations for controlling release of antipsychotic drug over prolonged periods of time which is highly beneficial. $\mathrm{MDT}_{30}$ ranged from 48.94 to $100.81 \mathrm{~h}$ thereby confirming the extended CPZ release potential of all formulations of the experimental design (Table 2). Greater $\mathrm{MDT}_{30}$ values corresponded with nanocapsule formulations that exhibited a faster CPZ release and is once again confirmed by Formulations 6 and 12. Possible strategies for controlling CPZ release further and counter-acting the burst release characteristics of the PCL-based nanocapsules revolve around 
incorporating the capsules with a polymeric matrix. In this case $\mathrm{CPZ}$ release would be separated into two phases: (1) diffusion of CPZ-loaded nanocapsules from the polymeric matrix upon hydration, and (2) dissolution of the nanocapsules causing CPZ release.
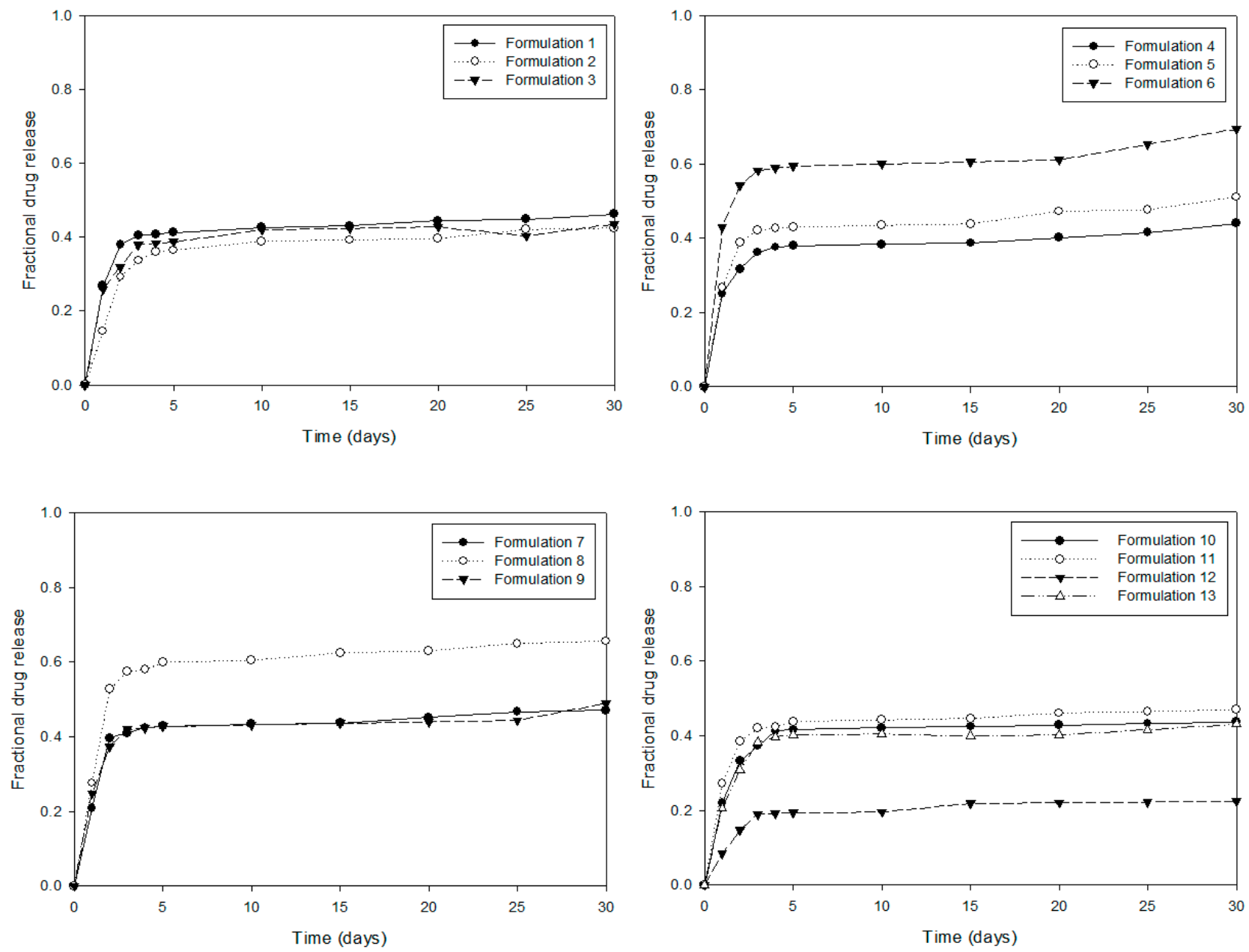

Figure 10. In vitro $\mathrm{CPZ}$ release profiles from PCL based nanocapsules as per experimental design, $(\mathrm{SD} \leq 0.04$ in all cases, $N=3$ ).

Table 2. MDT 30 values achieved for each formulation of the experimental design.

\begin{tabular}{cc}
\hline Formulation number & MDT $_{\mathbf{3 0}}$ (Hours) \\
\hline 1 & 66.23 \\
2 & 82.46 \\
3 & 60.79 \\
4 & 94.13 \\
5 & 100.81 \\
6 & 97.87 \\
7 & 65.12 \\
8 & 63.73 \\
9 & 95.93 \\
10 & 48.94 \\
11 & 52.23 \\
12 & 74.66 \\
13 & 68.59 \\
\hline
\end{tabular}




\subsection{Response Surface Analysis}

Response surface and contour plots (Figure 11) were derived for the measured responses (size, zeta potential and CPZ entrapment), based on the experimental model. Plots were used to represent the functional relationship between the experimental variables and the responses achieved. The effects of PCL and Polysorbate 80 on nanocapsule size are depicted in Figure 11a. It can be seen that low levels of PCL (1000-2000 mg) results in smaller capsules and vice versa. It is also demonstrated that increasing levels of Polysorbate 80 resulted in increases in capsules size.
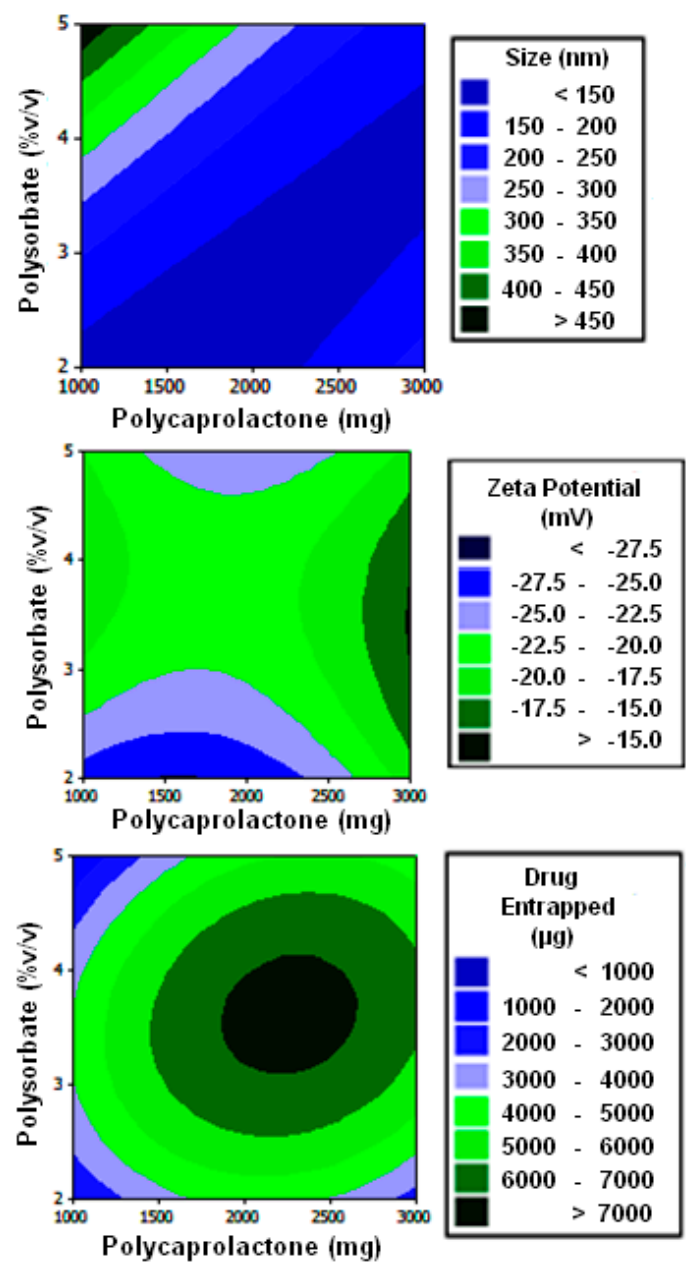

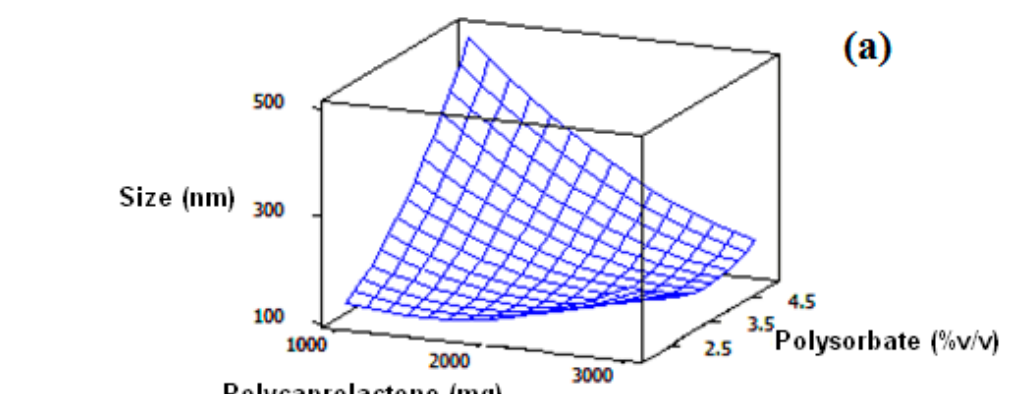

Polycaprolactone $(\mathrm{mg})$

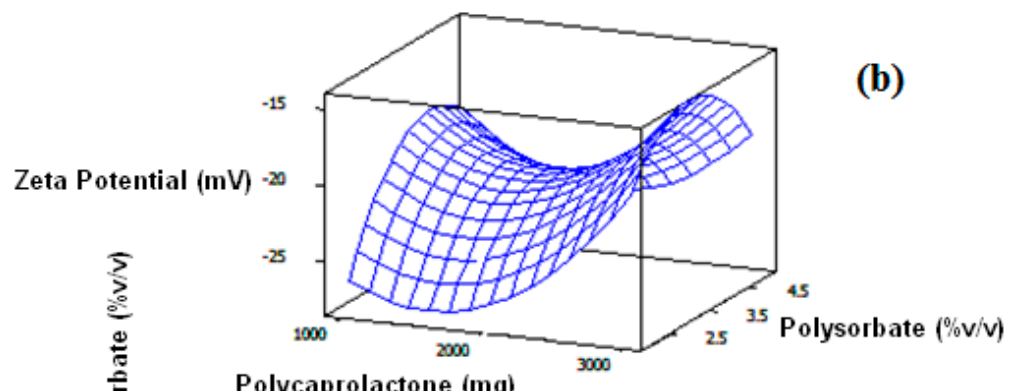

Polycaprolactone (mg)

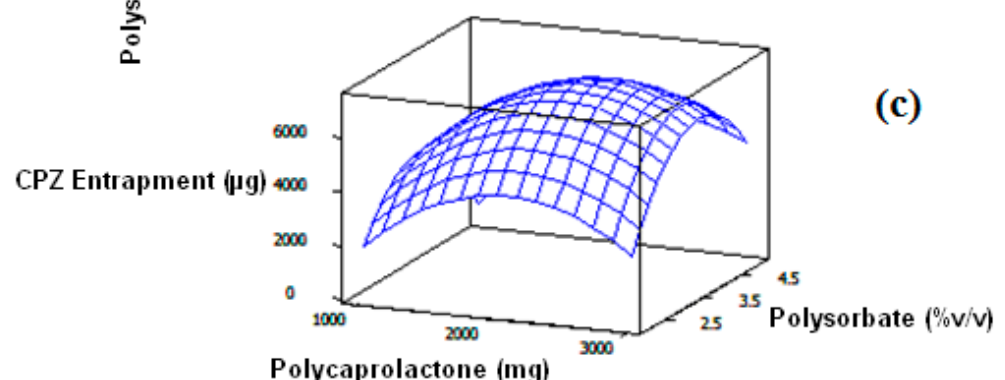

Polycaprolactone (mg)

Figure 11. Response surface and contour plots depicting the effects of PCL and Polysorbate 80 on (a) size, (b) zeta potential and (c) CPZ entrapment.

This is a typical result as generally, increasing the concentrations of the polymer and Polysorbate 80 does result in an increase in bulk volume of the dispersed phase thereby resulting in increased sizes of nanocapsules produced. The effects of PCL and Polysorbate 80 on zeta potential are demonstrated in Figure 11b. Lower levels of PCL (1000-2000 mg) resulted in more negative, more desirable zeta potential absolute values. Interactively, the most desirable zeta potentials were observed at lower PCL levels and low Polysorbate 80 concentration $(2.5 \% \mathrm{w} / \mathrm{v})$. This level of polymer to surfactant was thus the optimal range for enhancing the dispersion stability of the nanocapsules, enabling sufficient Polysorbate 80 absorption onto the PCL nanocapsule surface. High levels of PCL (3000 mg) in combination with 
median levels of Polysorbate $80(3.5 \% \mathrm{w} / \mathrm{v})$ produced the least favourable stabilisation of the nanocapsule system. The effects of PCL and Polysorbate 80 on CPZ entrapment are shown in Figure 11c. It can be seen that lower PCL values $(1000 \mathrm{mg})$ resulted in lower CPZ entrapment. At the same time, it can also be seen that high PCL values (3000 mg) resulted in low CPZ entrapment. A similar trend was observed with the effects of the levels of Polysorbate 80 on CPZ entrapment. The combined effects of PCL and Polysorbate 80 on the $\mathrm{CPZ}$ entrapment illustrate that $\mathrm{CPZ}$ entrapment is at a maximum when PCL levels are median and Polysorbate 80 levels are intermediate.

\subsection{Formulation Response Optimization}

A single, optimal formulation was developed subsequent to constraint optimization of nanocapsule size, zeta potential and CPZ entrapment. Response optimization was carried out utilizing statistical software (Minitab ${ }^{\circledR}$, V14, Minitab Inc. ${ }^{\circledR}$, State College, PA, USA) to determine the optimum level of each of PCL and Polysorbate 80 . Figure 12 depicts the desirability plots of each constraint for the single optimal formulation. Constraint settings utilized are represented in Table 3. The optimal levels of the independent variables that would achieve the desired nanocapsule size, zeta potential and CPZ entrapment are depicted in Table 4.

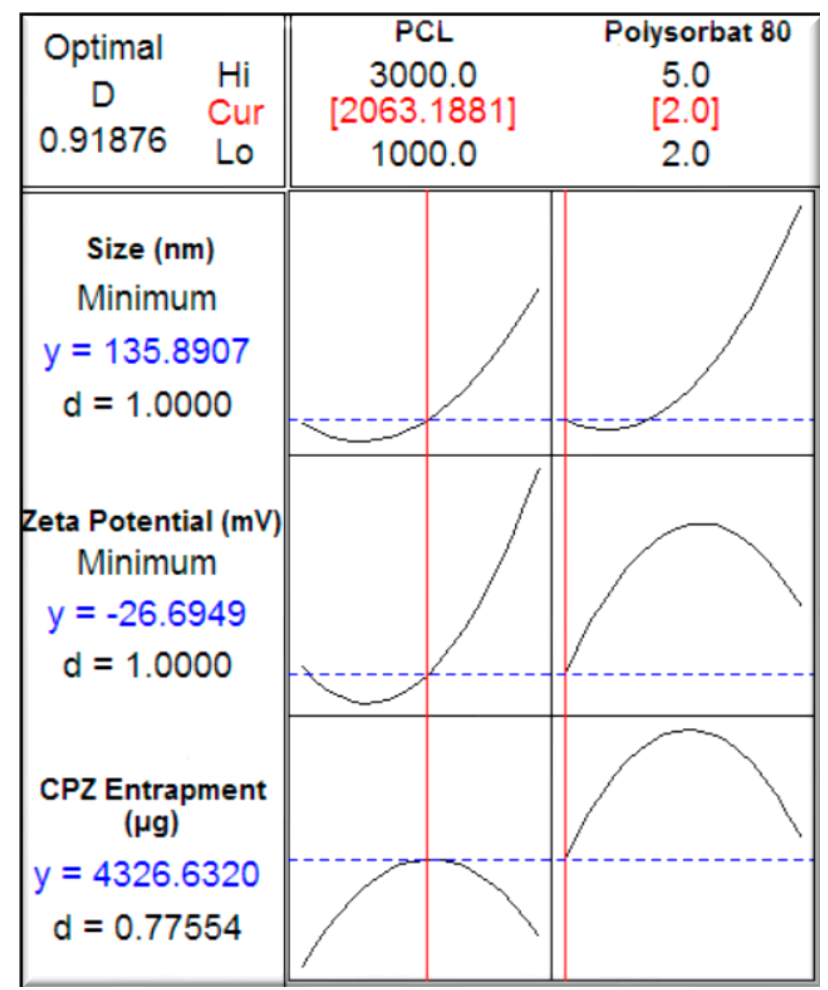

Figure 12. Desirability plots representing the levels of PCL and Polysorbate 80 required to synthesize the optimized formulation.

Table 3. Formulation constraints utilized for response optimization.

\begin{tabular}{cc}
\hline Responses & Limits \\
\hline Size & Minimize \\
Zeta Potential & Minimize \\
$\mathrm{CPZ}$ entrapment & Maximize \\
\hline
\end{tabular}


Table 4. Optimized CPZ-loaded, PCL based nanocapsule formulation derived from the response surface method.

\begin{tabular}{ccc}
\hline & PCL $(\mathbf{m g})$ & Polysorbate 80 (\% v/v) \\
\hline Optimized Formulation & 2063.1881 & 2 \\
\hline
\end{tabular}

Table 5 represents the optimized levels of the independent variables, the predicted responses, the desirability score as well as the correlation co-efficient for each response. The experimentally derived values for both size and zeta potential were in close agreement with predicted values of the statistical design with desirability being $96.9 \%$ and $98.9 \%$ respectively. Figure 13 depicts the distribution profiles for Figure 13a size and Figure $13 \mathrm{~b}$ zeta potential of the optimized formulation. Once again, the single peaks are representative of the limited distribution in size and zeta potential which are desirable. The experimental value achieved for CPZ entrapment was significantly higher than that of the predicted value and this exhibited a desirability of 53\%. Despite the low desirability of CPZ entrapment according to the experimental design, $\mathrm{CPZ}$ entrapment was significantly larger than predicted and did not result in larger nanocapsule sizes or drastic changes to zeta potential and stability. This therefore makes the CPZ entrapment and the experimental design in general advantageous for practical purposes.

Table 5. Predicted, experimental and desirability values of the CPZ-Loaded, PCL based nanocapsule formulation.

\begin{tabular}{cccc}
\hline Measured response & Predicted Value & Actual Value & Desirability (\%) \\
\hline Size $(\mathrm{nm})$ & 135.9 & $140.3 \pm 3.39$ & 96.9 \\
Zeta potential $(\mathrm{mV})$ & -26.7 & $-26.4 \pm 2.11$ & 98.9 \\
CPZ entrapment (\%) & 4326.6 & $8170.2 \pm 173.39$ & 53.0 \\
\hline
\end{tabular}
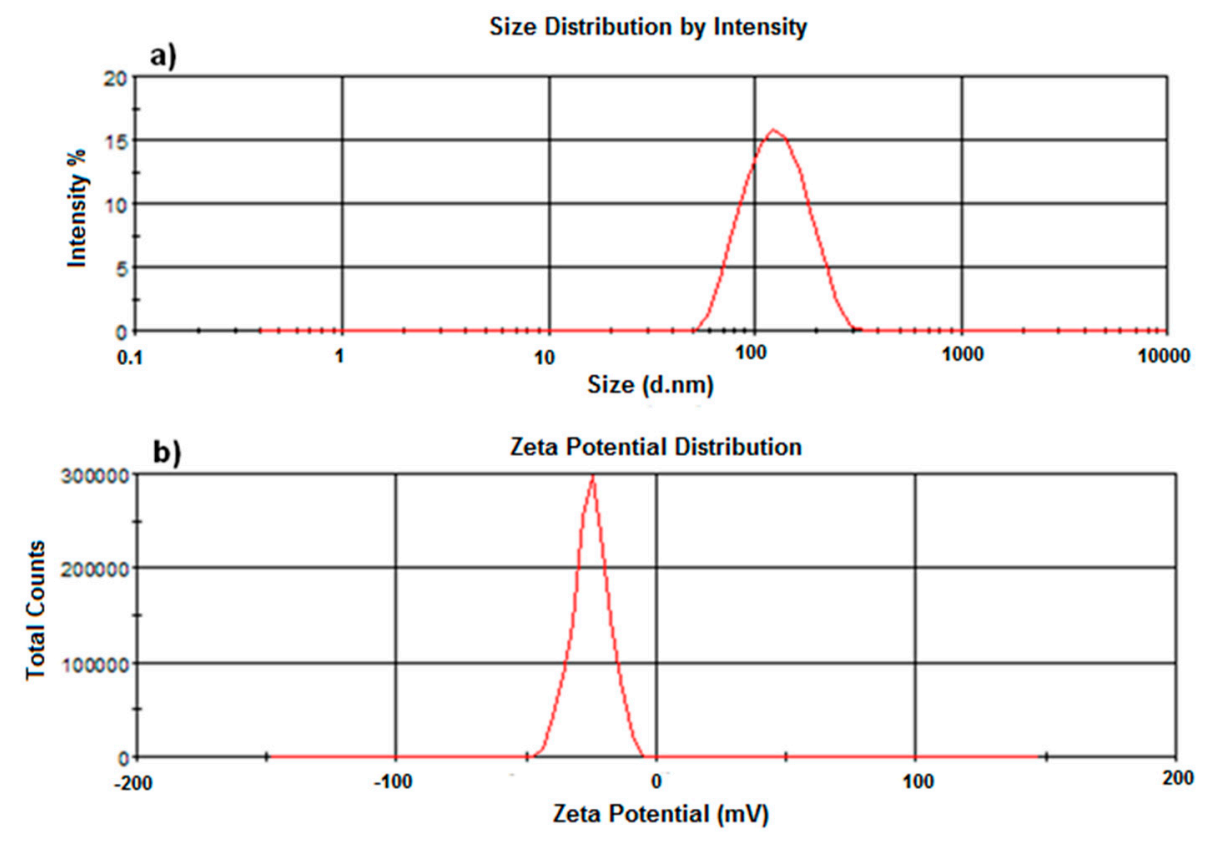

Figure 13. Distribution profiles of (a) size and (b) zeta potential of the optimized CPZ-loaded, PCL bases nanocapsule formulation. 
In addition, the yield and MDT 30 values of the optimized formulation were calculated to be $98.2 \%$ and $62.18 \mathrm{~h}$, respectively, and proved to be desirable (Table 6). Figure 14 is the CPZ release profile of the optimized nanocapsule formulation. A relatively high burst release of $42.0 \%$ of the entrapped CPZ is observed. This is justified by the relatively diminutive size (larger surface area) and high CPZ loading percentage. This is followed by the controlled release of CPZ and $45.6 \%$ of the entrapped drug is released after 30 days.

Table 6. Yield and MDT30 achieved for the optimized CPZ-loaded, PCL bases nanocapsule formulation.

\begin{tabular}{ccc}
\hline & Yield (\%) & MDT $_{\mathbf{3 0}}$ \\
\hline Optimized Formulation & $98.2 \pm 1.25$ & $67.14 \pm 9.91$ \\
\hline
\end{tabular}

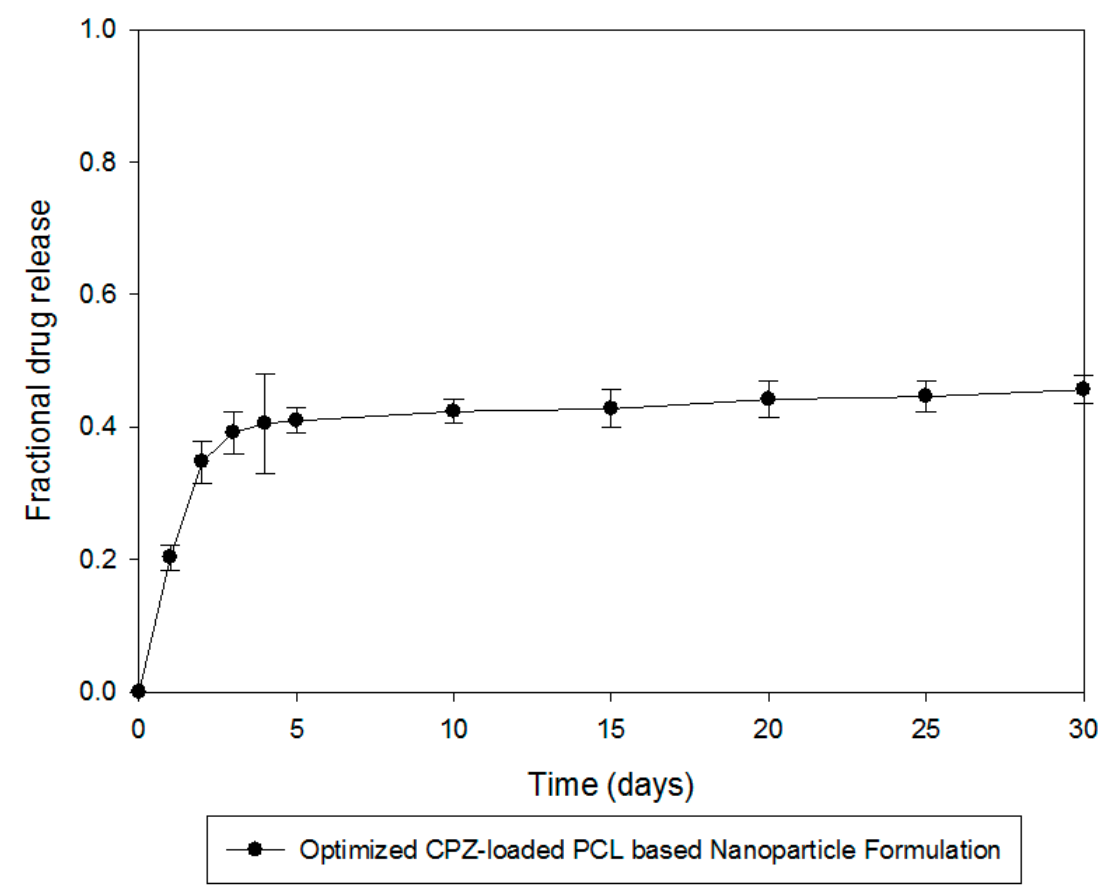

Figure 14. CPZ release from the optimized CPZ-loaded, PCL based nanocapsule formulation.

\subsection{In Vitro Cytotoxicity Analysis in a Neuronal Cell Line}

Light microscopy was utilized to assess the growth progression of the PC12 neuronal cells (Figure 15). Figure 15a,b represents cell growth after three days at a magnification of $40 \times$ and Figure $15 \mathrm{c}$ represents cell growth progression after 7 days at $25 \times$ magnification. Images reveal a highly proliferative cell growth at 7 days. Furthermore, healthy cells were observed microscopically as there was no visible lysis and cells were intact.

After a $48 \mathrm{~h}$ incubation period with the respective controls, nanocapsules and components, the cell viability in terms of their death relative to the number of cells originally incubated with the respective compounds, was assessed and is depicted in Figure 16. The PCL, CPZ, and CPZ-free and loaded nanocapsules possessed insignificant cytotoxic effects compared to the positive control $(p>0.05)$. The low toxicity of the optimal nanocapsule formulation also indicates that its components, including Polysorbate 80 , were minimally toxic to neuronal cells at the level employed. 


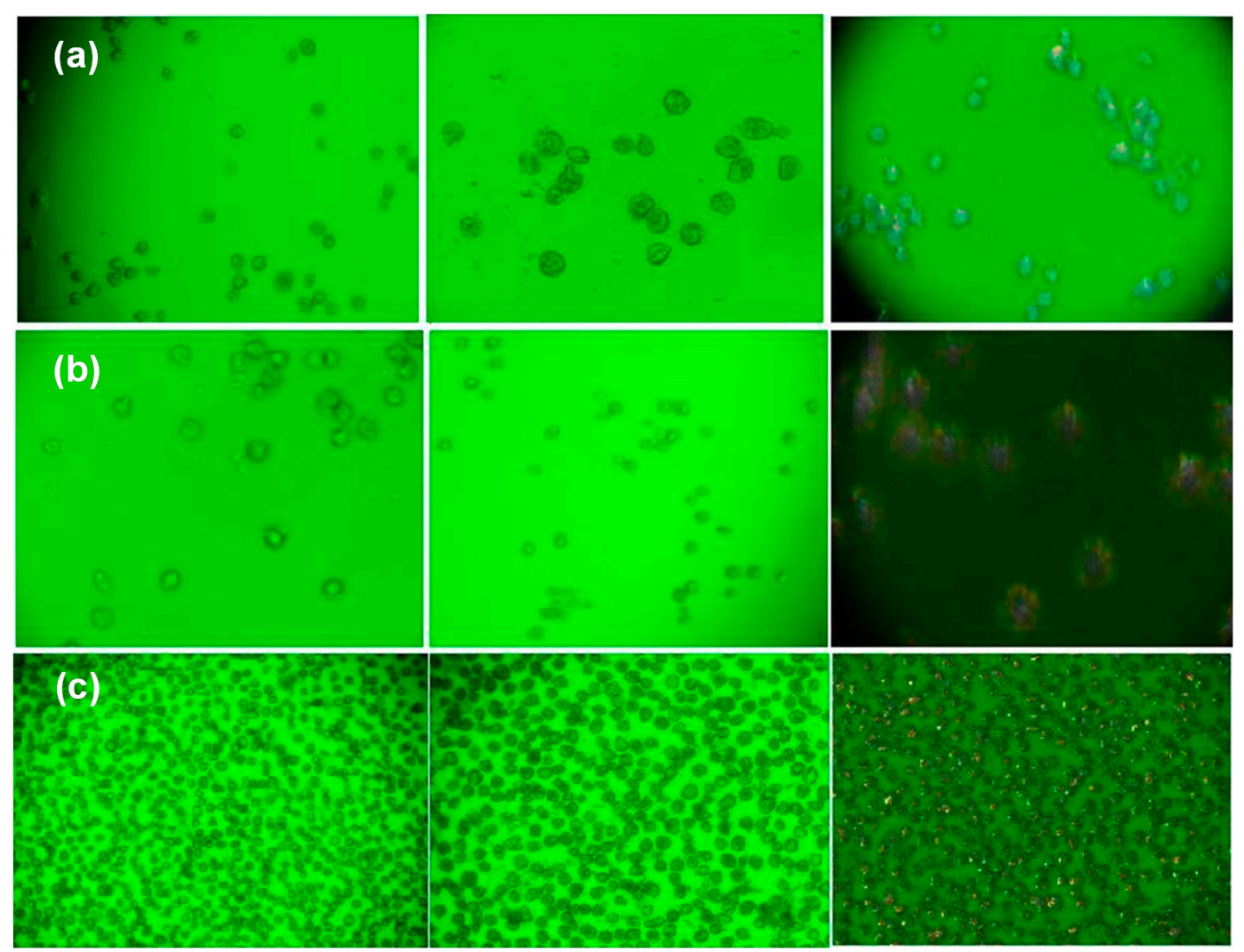

Figure 15. Light microscopy images of PC12 neuronal cells at (a) and (b) 3 days at 40× magnification and (c) 7 days at $25 \times$ magnification.

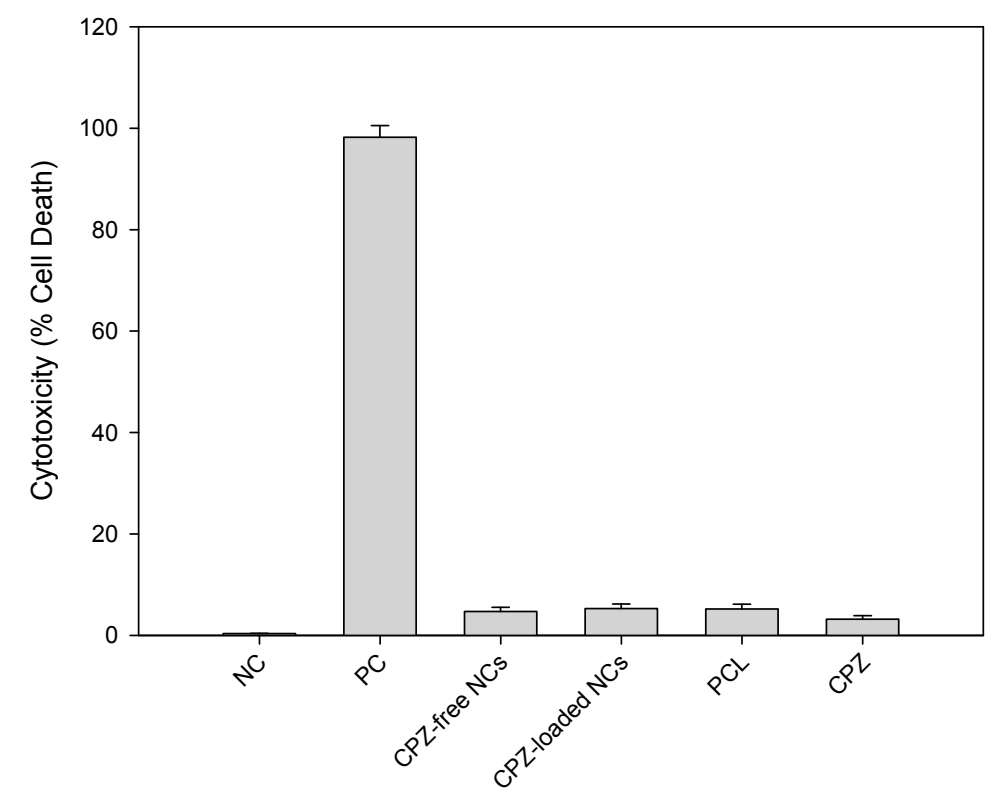

Figure 16. Cytotoxic evaluation of PC12 neuronal cells upon exposure to the nanocapsules utilizing the CytoTox $96^{\circledR}$ Non-Radioactive Cytotoxicity Assay.

\subsection{In Vivo Analysis of the Nanocapsule-Loaded Polymeric Membrane in a Rabbit Model}

Figure 17 depicts the optimized nanocapsules incorporated within an implantable polymeric membrane system (NCPM) prepared for implantation in the frontal lobe of the rabbit brain to achieve site-specific 
prolonged $\mathrm{CPZ}$ delivery within the therapeutic range for a prolonged period to assess its potential for the enhanced management of psychotropic disorders Figure 18 outlines and summarizes the entire implantation procedure.

$\mathrm{H} \& \mathrm{E}$ staining and histopathology of the brain implanted with NCPM (drug-loaded and drug-free), the non-drug loaded NCPM revealed a superficial subdural area of liquifactive necrosis which most like due to the implantation of the NCPM (also observed for the drug-free membrane) and the subsequent displacement of the neuroparenchyma by the implant with mild inflammation present around the necrotic area. The abundance of macrophages or glitter cells are indicative of healing and phagocytosis of the necrotic brain material. The mild inflammation is only limited to the region of the implant and does not extend widely throughout the brain. Furthermore, minimal hemorrhaging is observed. These results confirm the success of the implantation procedure and confirm the biocompatibility of the NCPM.

The in vivo CPZ release profiles from the NCPM and the stat dose of the commercially available Chlorpromazine HCl-Fresenius ${ }^{\circledR} 25 \mathrm{mg} / \mathrm{mL}$ administered intramuscularly are displayed in Figure 19. It is noted that the NCPM and the stat dose of the commercially available Chlorpromazine HCl-Fresenius ${ }^{\circledR}$ $25 \mathrm{mg} / \mathrm{mL}$ display contrasting results. The release profiles from the NCPM displayed higher levels of CPZ in the CSF when compared to the commercially available Chlorpromazine HCl-Fresenius ${ }^{\circledR}$ $25 \mathrm{mg} / \mathrm{mL}$ injection which displayed higher CPZ levels in the plasma when compared to the CSF. Furthermore, the CPZ peak levels $\left(C_{\max }=2.92 \mathrm{ng} / \mathrm{mL}\right)$ from the NCPM are reached after 7 days following implantation and thereafter, $\mathrm{CPZ}$ is released in a controlled manner. No irregular fluctuations of CPZ are noted from the NCPM which is highly desirable. A plasma $C_{\max }$ value of $0.95 \mathrm{ng} / \mathrm{mL}$ was detected at Day 3 following implantation with the NCPM. The CPZ detected in the blood could possibly be attributed to the normal CSF flow and turnover, in which substances enter the venous blood flow via the arachnoid granulations [22]. This theory is highly substantiated as the size of the nanoparticles which would make them much more susceptible to passing through the arachnoid granulations and into the systemic circulation.

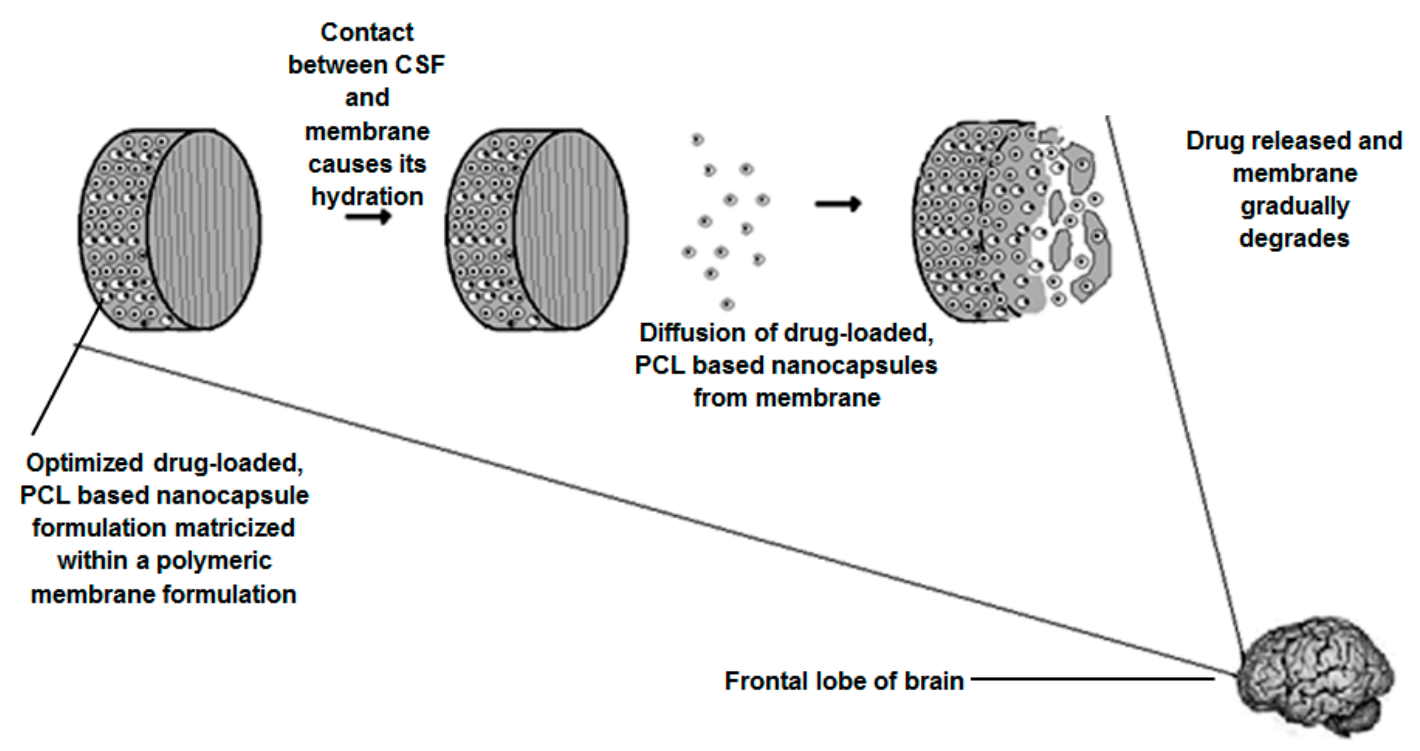

Figure 17. Proposed mechanism of action and site of implantation of the optimized nanocapsules incorporated within a polymeric membrane. 


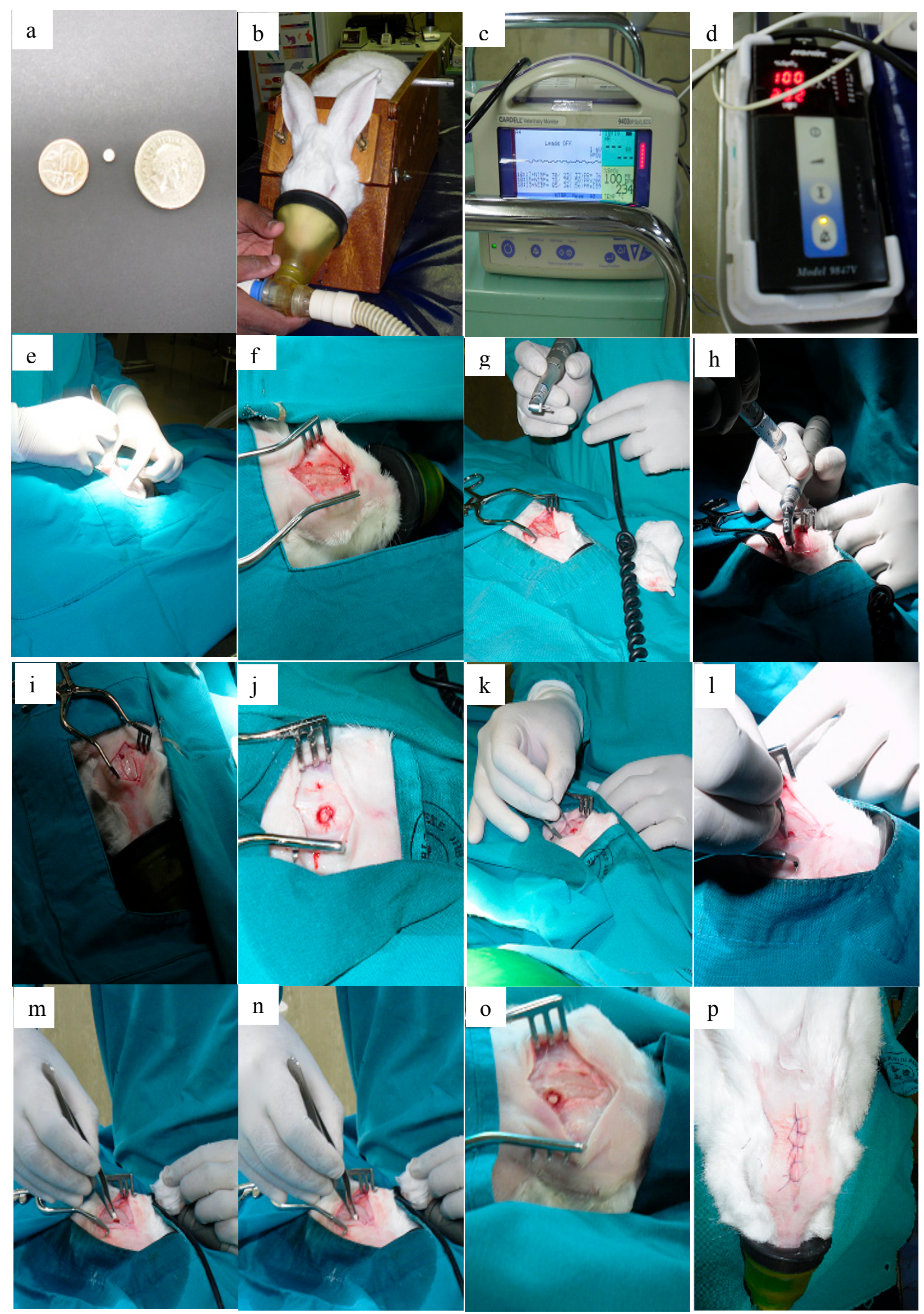

Figure 18. Implantation of the NCPM into the frontal lobe of the New Zealand White Rabbit brain (Time frame $=15 \mathrm{~min}$ ). (a) Relative size of the implanted NCPM when compared to a 
Great Britain Pound (GBP) and a South African 10 cent coin; (b) Anesthesia of rabbits with a mixture of Ketamine $(50 \mathrm{mg} / \mathrm{kg})$ and Xylazine $(5 \mathrm{mg} / \mathrm{kg})$. (c,d) Monitoring of vital signs of the rabbit such as heart rate, respiratory rate, temperature, etc. during surgery; (e) Midline incision made and the skull is exposed; (f,g) Skull is exposed and land-marking sutures are clearly visible; (h) A dental drill is used to drill through the skull and pierce the dura mater with minimal bleeding and the area is flushed with saline; (i,j) A clearly visible unilateral burrhole $(5 \mathrm{~mm})$ is made anterior to the coronal suture and lateral to the saggital suture; $(\mathbf{k}, \mathbf{l})$ The cortex and parenchyma of the brain is pierced through the burrhole; $(\mathbf{m}, \mathbf{o})$ The NCPM is placed inside the parenchyma and is clearly visible; (p) The scalp insertion is closed with non-absorbable suture while the rabbit is maintained on anesthetic.
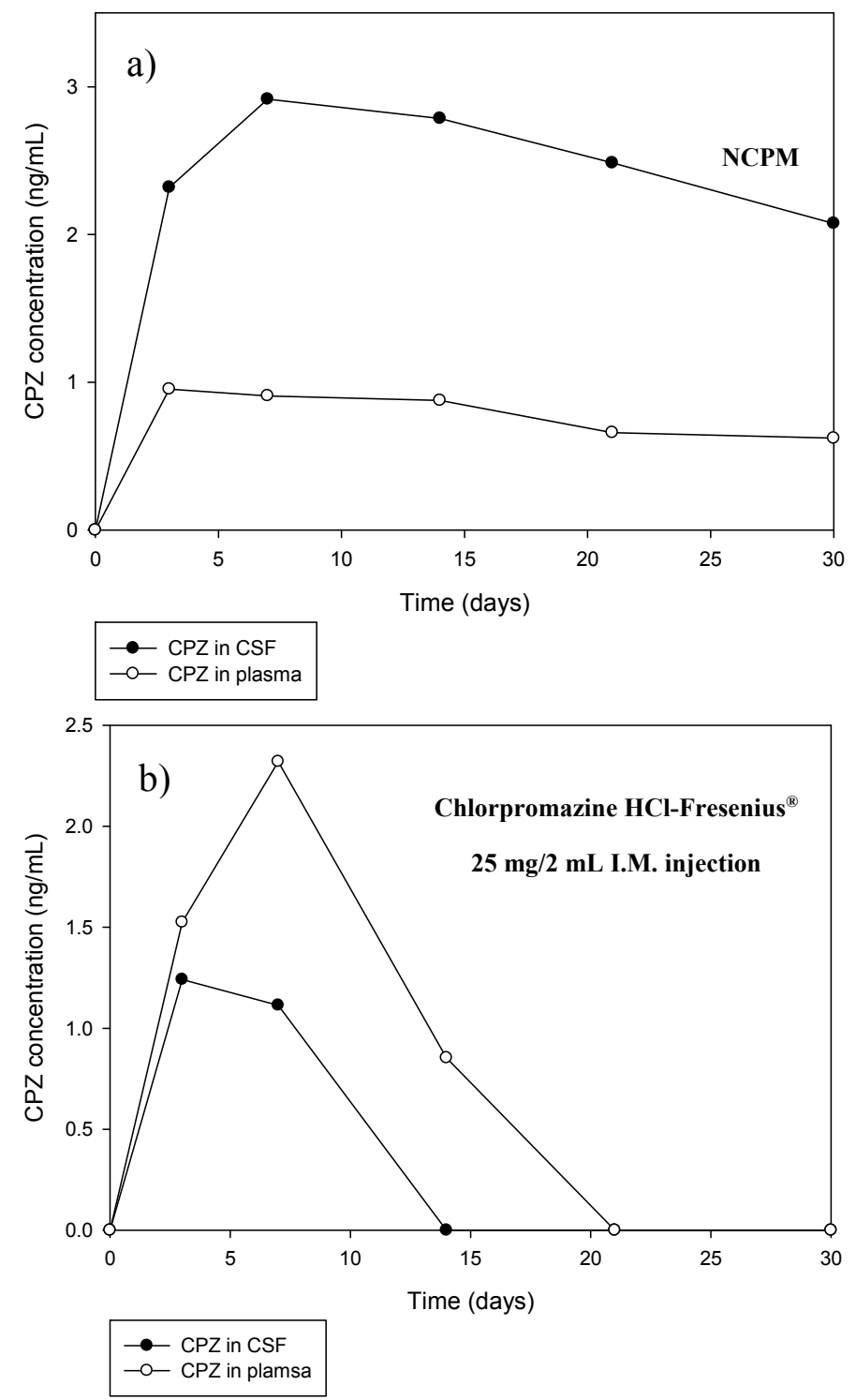

Figure 19. In vivo $\mathrm{CPZ}$ release from (a) the NCPM and (b) the commercially available Chlorpromazine $\mathrm{HCl}$-Fresenius ${ }^{\circledR} 25 \mathrm{mg} / \mathrm{mL}$ ( $\mathrm{SD} \leq 0.31$ in all cases).

The intramuscular dose of CPZ (Chlorpromazine HCl-Fresenius ${ }^{\circledR} 25 \mathrm{mg} / \mathrm{mL}$ ) revealed higher levels of CPZ in the blood plasma when compared to the CSF. This is due to the extremely low bioavailability 
of CPZ (approximately 20\% plasma bioavailability due to high first-pass metabolism). A $C_{\max }$ value of $1.24 \mathrm{ng} / \mathrm{mL}$ was computed in CSF after Day 3 following the intramuscular injection and a $C_{\max }$ value of $2.32 \mathrm{ng} / \mathrm{mL}$ was determined in plasma at Day 7 following intramuscular administration of the commercially available Chlorpromazine HCl-Fresenius ${ }^{\circledR} 25 \mathrm{mg} / \mathrm{mL}$ injection. CPZ could only be detected in the blood plasma up until Day 14 and the CSF up until Day 7. These results are very atypical and surprising because $\mathrm{CPZ}$ in the tablet or injectable forms are dosed up to three times a day. A reason for the CPZ detection in the blood and CSF could possibly be due to the fact that the drug is highly protein bound and may attach itself to the surrounding fatty tissue [32].

The CPZ release from the NCPM was far superior to the commercially available form of the drug. Implantation at the site of action allowed for a drastic increase in the CPZ bioavailability. Furthermore, the drug was released in a pseudo-steady state with CSF levels being maintained between 2.00 and $3.00 \mathrm{ng} / \mathrm{mL}$ with no fluctuations observed. CPZ levels in the CSF and plasma attained from the commercially available Chlorpromazine HCl-Fresenius ${ }^{\circledR} 25 \mathrm{mg} / \mathrm{mL}$ injection displayed a high degree of fluctuation in both CSF $(1.11-1.24 \mathrm{ng} / \mathrm{mL})$ and plasma $(0.85-2.32 \mathrm{ng} / \mathrm{mL})$. An area of concern is the fact that $\mathrm{CPZ}$ is being cleared into the blood. This could result in systemic side-effects. The fact that the visual assessment of the rabbits during the course of the study revealed that the rabbits experienced no visible signs of discomfort or abnormal behavior (including posture, movement) may suggest that the doses entering the systemic circulation are not significant enough to cause any side-effects.

\section{Conclusions}

The novel melt-dispersion technique for nanocapsule synthesis proved to be conducted with relative ease and confirmed the success of entrapping the antipsychotic drug, CPZ. Furthermore, the technique did not require the use of physiologically and environmentally toxic organic solvents was a relatively inexpensive procedure and was devoid of any major complications. The use of thermoplastic polymers that are not easily degraded by increased temperatures is a limitation of the melt dispersion technique as is the use of highly hydrophilic drugs resulting in low entrapment. Despite this, extensive in vitro testing and optimization, with subsequent incorporation of the nanocapsules in an implantable membrane with in vivo testing in a rabbit model, supports the application for the use and feasibility of the CPZ-loaded, PCL based nanocapsules for the long-term management of certain psychotropic disorders where the benefits of nanotechnology can be exploited.

\section{Acknowledgments}

This work was funded by the National Research Foundation (NRF) of South Africa and the Gauteng Department of Agriculture and Rural Development (GDARD) of South Africa.

\section{Author Contributions}

Thiresen Govender, Yahya E. Choonara, Lisa C. du Toit, Girish Modi and Viness Pillay conceptualized the study. Thiresen Govender conducted the in vitro and in vivo research. Dinesh Naidoo assisted with in vivo studies. Lisa C. du Toit and Pradeep Kumar assisted with in vitro studies and drafted the manuscript. All authors read and approved the final manuscript. Viness Pillay is the corresponding author. 


\section{Conflicts of Interest}

The authors declare that there are no conflicts of interest.

\section{References}

1. Mak, M.; Fung, L.; Strasser, J.F.; Saltzman, W.M. Distribution of drugs following controlled delivery to the brain interstitium. J. Neuro-Oncol. 1995, 26, 91-102.

2. Pardridge, W.M. Drug delivery to the brain. J. Cereb. Blood Flow Metab. 1997, 17, 713-731.

3. Pardridge, W.M. The blood-brain barrier: Bottleneck in brain drug development. NeuroRX 2005, 2, 3-14.

4. Bodor, N.; Buchwald, P. Brain-targeted drug delivery: Experiences to date. Am. J. Drug Deliv. 2003, 1, 13-26.

5. Unger, E.C.; Porter, T.; Culp, W.; Labell, R.; Matsunaga, T.; Zutshi, R. Therapeutic applications of lipid-coated microbubbles. Adv. Drug Deliv. Rev. 2004, 56, 1291-1314.

6. Krauze, M.T.; Forsayeth, J.; Park, J.W.; Bankiewicz, K.S. Real-time imaging and quantification of brain delivery of liposomes. Pharm. Res. 2006, 23, 2493-2504.

7. Pardridge, W.M. Why is the global CNS pharmaceutical market so underpenetrated? Drug Discov. Today 2002, 7, 5-7.

8. Wohlfart, S.; Gelperina, S.; Kreuter, J. Transport of drugs across the blood-brain barrier by nanoparticles. J. Control. Release 2011, 161, 264-273.

9. Manjunath, K.; Venkateswarlu, V. Pharmacokinetics, tissue distribution and bioavailability of clozapine solid lipid nanoparticles after intravenous and intraduodenal administration. J. Control. Release 2005, 107, 215-228.

10. Muthu, M.S.; Rawat, M.K.; Mishra, A.; Singh, S. PLGA nanoparticle formulations of risperidone: preparation and neuropharmacological evaluation. Nanomed. Nanotechnol. Biol. Med. 2009, 5, 323-333.

11. Benvegnú, D.M.; Barcelos, R.C.S.; Boufleur, N.; Reckziegel, P.; Pase, C.S.; Ourique, A.F.; Beck, R.C.R.; Bürger, M.E. Haloperidol-loaded polysorbate-coated polymeric nanocapsules increase its efficacy in the antipsychotic treatment in rats. Eur. J. Pharm. Biopharm. 2011, 77, 332-336.

12. Seju, U.; Kumar, A.; Sawant, K.K. Development and evaluation of olanzapine-loaded PLGA nanoparticles for nose-to-brain delivery: In vitro and in vivo studies. Acta Biomater. 2011, 7, 4169-4176.

13. Silva, A.C.; González-Mirac, E.; Garcíac, M.L.; Egeac, M.A.; Fonsecaa, J.; Silva, R.; Santos, D.; Souto, E.B.; Ferreira, D. Preparation, characterization and biocompatibility studies on risperidone-loaded solid lipid nanoparticles (SLN): High pressure homogenization versus ultrasound. Colloid Surf. B Biointerf. 2011, 86, 158-165.

14. Soppimath, K.S.; Aminabhavi, T.M.; Kulkarni, A.R.; Rudzinski, W.E. Biodegradable polymeric nanoparticles as drug delivery devices. J. Control. Release 2001, 70, 1-20.

15. Hans, M.L.; Lowman, A.M. Biodegradable nanoparticles for drug delivery and targeting. Curr. Opin. Solid State Mater. Sci. 2002, 6, 319-327.

16. Reis, C.P.; Neufeld, R.J.; Ribeiro, A.J.; Veiga, F. Nanoencapsulation I. Methods for preparation of drug-loaded polymeric nanoparticles. Nanomed. Nanotechnol. Biol. Med. 2006, 2, 8-21. 
17. Sinha, V.R.; Bansal, K.; Kaushik, R.; Kumria, R.; Trehan, A. Poly-e-caprolactone microspheres and nanospheres: An overview. Int. J. Pharm. 2004, 278, 1-23.

18. Wei, X.W.; Gong, C.Y.; Gou, M.L.; Fu, S.Z.; Guo, Q.F.; Shi, S.; Luo, F.; Guo, G.; Qiu, L.Y.; Qian, Z.Y. Biodegradable poly(ع-caprolactone)-poly(ethylene glycol) copolymers as drug delivery system. Int. J. Pharm. 2009, 381, 1-18.

19. Kumari, A.; Yadav, S.K.; Yadav, S.C. Biodegradable polymeric nanoparticles based drug delivery systems. Colloid Surf. B Biointerf. 2010, 75, 1-18.

20. Kolowale, O.A.; Pillay, V.; Choonara, Y.E. Novel polyamide 6,10 variants synthesized by modified interfacial polymerization for application as a rate-modulated monolithic drug delivery system. J. Bioact. Compat. Polym. 2007, 22, 281-313.

21. Brem, H.; Kader, A.; Epstein, J.I.; Tamargo, R.J.; Domb, A.; Langer, R.; Leong, K.W. Biocompatibility of a biodegradable controlled-release polymer in the rabbit brain. Select. Canc. Ther. 1989, 5, 55-65.

22. Grzybowski, D.M.; Holman, D.W.; Katz, S.E.; Lubow, M. In vitro model of cerebrospinal fluid outflow through human arachnoid granulations. Invest. Ophthal. Vis. Sci. 2006, 47, 3664-3672.

23. Elzein, T.; Nasser-Eddine, M.; Delaite, C.; Bistac, S.; Dumas, P. FTIR study of polycaprolactone chain organization at interfaces. J. Colloid. Interface Sci. 2004, 273, 381-387.

24. Wu, C. Analysis of mechanical, thermal, and morphological behavior of polycaprolactone/wood flour blends. J. Appl. Polym. Sci. 2004, 94, 1000-1006.

25. Khan, Y.; Durrani, S.K.; Mehmooda, M.; Ahmada, J.; Khan, M.R.; Firdous, S. Low temperature synthesis of fluorescent $\mathrm{ZnO}$ nanoparticles. Appl. Surf. Sci. 2010, 257, 1756-1761.

26. Zhao, Q.; Tao, J.; Yam, R.C.M.; Mok, A.C.K.; Li, R.K.Y.; Song, C. Biodegradation behavior of polycaprolactone/rice husk ecocomposites in simulated soil medium. Polym. Degrad. Stab. 2008, 93, 1571-1576.

27. Mohanraj, V.J.; Chen, Y. Nanoparticles-A review. Trop. J. Pharm. Res. 2006, 5, 561-573.

28. Kato, H.; Mizuno, K.; Shimada, M.; Nakamura, A.; Takahashi, K.; Hata, K.; Kinugasa, S. Observations of bound Tween80 surfactant molecules on single-walled carbon nanotubes in an aqueous solution. Carbon 2009, 47, 3434-3440.

29. Ruckmani, K.; Sankar, V. Formulation and optimization of zidovudine niosomes. AAPS PharmSciTech 2010, 11, 1119-1127.

30. Midhun, B.T.; Shalumon, K.T.; Manzoor, K.; Jayakumar, R.; Nair, S.V.; Deepthy, M. Preparation of budesonide-loaded polycaprolactone nanobeads by electrospraying for controlled drug release. J. Biomater. Sci. 2011, 22, 2431-2444.

31. Chawla, J.S.; Amiji, M.M. Biodegradable poly(o-caprolactone) nanoparticles for tumortargeted delivery of tamoxifen. Int. J. Pharm. 2002, 249, 127-138.

32. Okeri, H.A.; Alonge, P.O.; Etareri, E. Comparitive determination of chlorpromazine hydrochloride content in multi-sourced chlorpromazine tablets in Nigeria. Int. J. Health Res. 2008, 1, 21-26.

(C) 2015 by the authors; licensee MDPI, Basel, Switzerland. This article is an open access article distributed under the terms and conditions of the Creative Commons Attribution license (http://creativecommons.org/licenses/by/4.0/). 